\title{
Lisfranc Injury Imaging and Surgical Management
}

\author{
Eva Llopis, $\mathrm{MD}^{1}$ Javier Carrascoso, $\mathrm{MD}^{2}$ Inigo Iriarte, $\mathrm{MD}^{3}$ Mariano de Prado Serrano, $\mathrm{MD}^{4}$ \\ Luis Cerezal, MD ${ }^{5}$
}

${ }^{1}$ Department of Radiology, Hospital de la Ribera, Alzira, Valencia,

Spain

Address for correspondence Eva Llopis, MD, Hospital de la Ribera,

2 Department of Radiology, Hospital Quiron, Madrid, Spain

Alzira, Valencia, Spain (e-mail: Evallopis@gmail.com).

${ }^{3}$ Department of Rehabilitation, Clinica Ars, Bilbao, Spain

${ }^{4}$ Department of Orthopedics, Hospital Quiron Murcia, Murcia, Spain

${ }^{5}$ Department of Radiology, Centro Diagnostico Médico Cantabria,

Santander, Spain

Semin Musculoskelet Radiol 2016;20:139-153.

\begin{abstract}
Keywords

- Lisfranc

- midfoot

- fracture dislocation
\end{abstract}

When Jacques Lisfranc served as a military surgeon in Napoleon's army, he described a quick forefoot amputation through the tarsometatarsal (TMT) joint to treat the extended dislocation that occurred when the foot was trapped in the stirrup falling from a horse. Although he did not describe any fracture or dislocation, many midfoot structures and injuries were subsequently named for Lisfranc including the TMT joint, the interosseous ligament between the first cuneiform bone and the base of the second metatarsal bone, and the spectrum of injuries along the TMT joints including their stabilizing ligamentous complexes. ${ }^{1-3}$

There are two different groups of injuries: those secondary to high-energy trauma, with fractures or fracture dislocation of the midfoot, and those secondary to low-energy trauma, with often subtle Lisfranc injuries or sprains. Lisfranc fractures and injuries are rare and frequently overlooked. Delayed diagnosis and treatment can lead to long-term pain and disability, secondary to early osteoarthritis (OA), flattening of the arch, chronic instability, and pain in the midfoot. ${ }^{2,3}$

Lisfranc injuries and fractures have been cited among the most common reasons for legal claims. The fact that these injuries may occur in the context of polytrauma and may be subtle explains why they are often overlooked. ${ }^{4,5}$

\section{Relevant Anatomy}

The Lisfranc joint, or TMT joint, is the transition between the rigid midfoot and the relatively flexible forefoot, and structurally it supports the transverse arch of the foot. It includes the joints between the cuneiforms and cuboid with the bases of the five metatarsal bones. Stability is achieved together with the intervening ligamentous structures. These form three different biomechanical and synovial compartments. The medial column or first ray is formed by the first cuneiform (C1) and first metatarsal (M1) and covered by the medial synovial membrane. The intermediate or middle column consists of the second and third ray, formed by the second and third cuneiform ( $\mathrm{C} 2$ and $\mathrm{C} 3$ ) with the corresponding second and third metatarsals (M2, M3); it is closely related to the cuneoscaphoid joint. The lateral column includes the cuboid, the fourth and fifth metatarsal (M4 and M5), comprising the fourth and fifth ray; its synovial membrane does
Issue Theme Imaging of the Midfoot and Forefoot; Guest Editor, Hilary Umans, MD
Copyright (C) 2016 by Thieme Medical Publishers, Inc., 333 Seventh Avenue, New York, NY 10001, USA.

Tel: +1(212) 584-4662.
DOI http://dx.doi.org/ 10.1055/s-0036-1581119. ISSN 1089-7860. 
not contact with the middle compartment. ${ }^{1,6,7}$ The lateral column is positioned more proximally with respect to the medial column ${ }^{6}$ (-Figs. 1 and $\mathbf{2}$ ).

\section{Bones}

Bones are the primary structural support. The cuneiform bones are wedge shaped and articulate with the first, second, and third metatarsal bases. The articular surfaces between $\mathrm{C} 1$ and M1 form a crescent shape. ${ }^{6}$ The middle cuneiform bone is located 4 to $8 \mathrm{~mm}$ proximal to the medial and lateral cuneiform, forming a recess into which the base of M2 locks. A developmentally shallower recess has been shown to predispose to injuries of the second ray. ${ }^{8}$

The bases of the M1, M2, and M3 bones, especially the second one, have a trapezoidal wedge shape, with a broad dorsal aspect and plantar apex; this trapezoidal shape predisposes to dorsal displacement. Usually the bases of M1 and M2 do not articulate. In some cases, however, there can be some contact between the lateral base of M1 and medial base of M2 with an occasional small articular facet at the medial base of M2. M2 and M3 each have two articular surfaces (-Fig. 2). 1,6,9

A vertical crest at the joint surface of the cuboid divides the medial and lateral facets that articulate with the fourth and fifth metatarsal bones, respectively. The lateral column, cuboid M4-M5, is the most mobile of the Lisfranc joint. ${ }^{6}$

The metatarsal bases, together with the cuneiform bones, form an arch resembling a roman arch, in which the C2-M2 joint represents the keystone for stability, preventing mediallateral and plantar displacement ${ }^{1,8,9}$ (-Figs. 1-3).

\section{Ligaments}

A complex ligamentous and capsule system maintains midfoot stability. There is extreme variability in the course, number, and insertions of the ligaments. ${ }^{6}$ MRI allows direct visualization of the ligaments using different scan planes and pulse sequences. The ligaments are structured based on their orientation: transverse, longitudinal, or oblique, and they have different components based on their location: dorsal, interosseous, or plantar (-Figs. 4-9). Transverse ligaments connect adjacent bones: intertarsal, intercuneiform, and intermetatarsal. No ligament connects the first and second metatarsals. Longitudinal ligaments extend from the tarsal to the metatarsal bones. Oblique ligaments connect adjacent rays; the most important component is the Lisfranc ligament complex coursing from $\mathrm{C} 1$ to $\mathrm{M} 2$. $^{1,3}$

The three types of dorsal ligaments are the intertarsal (cuneiforms and cuboid bones), intermetatarsal, and those connecting cuneiforms to the metatarsal. The last includes three short ligaments from the cuneiforms to the base of the M2 that are visible in the oblique sagittal plane as a thin low signal intensity (SI) band. ${ }^{1,6}$ The dorsal ligaments are short and flat and the weakest of the ligamentous complex. Because the dorsal ligaments are more prone to rupture, there is a tendency for dorsal dislocation of the metatarsal bases. ${ }^{2,8}$

The interosseous ligaments are thick and strong: three intermetatarsal ligaments (between the bases from M2 to M5), three intertarsal ligaments (between the cuneiforms),

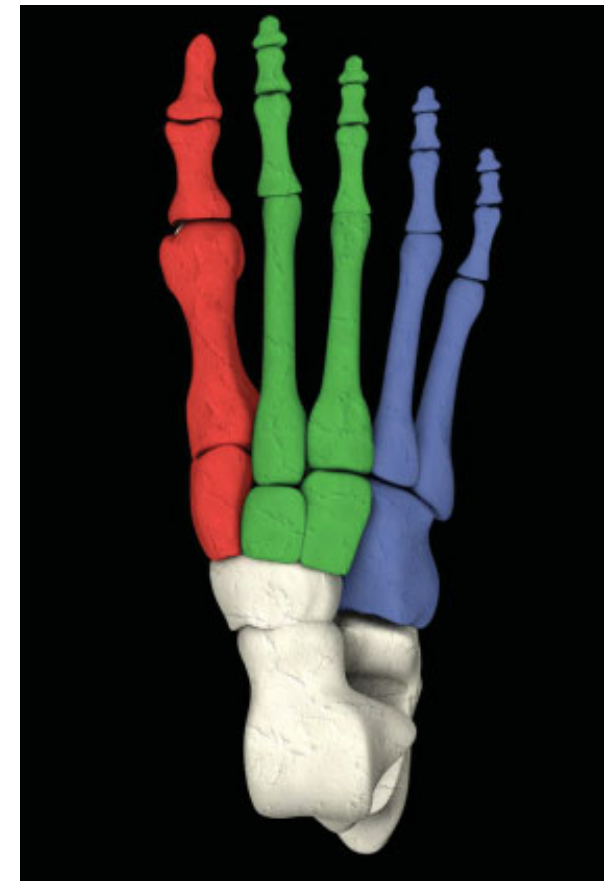

Fig. 1 Midfoot is divided into three independent columns, medial (red), middle or intermediate (green), and lateral (blue).

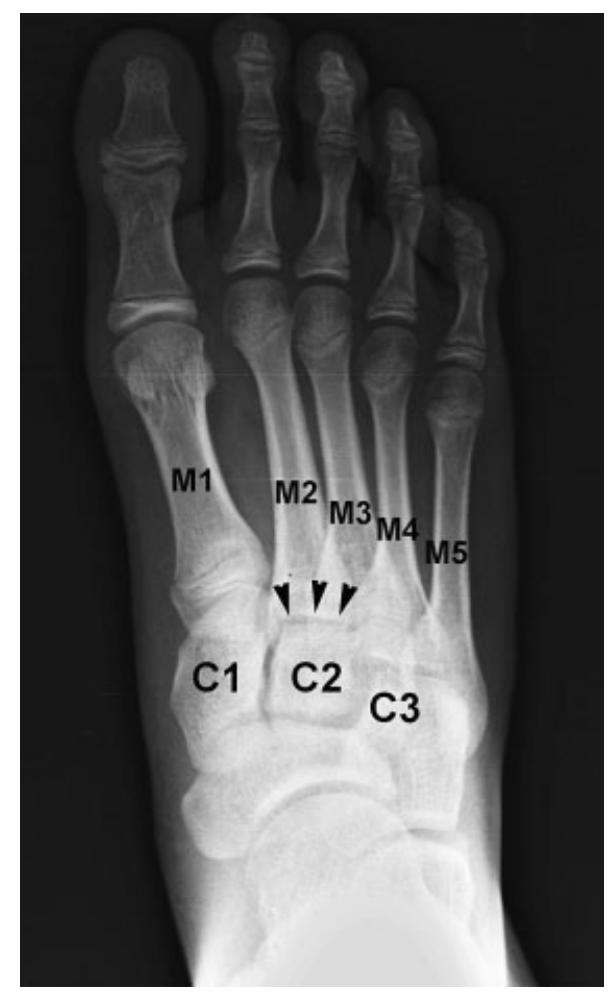

Fig. 2 Normal anteroposterior plain x-ray of the foot. The Lisfranc joint is the joint between the cuneiform bones, medial (C1), middle (C2), and lateral (C3), and the cuboid and the metatarsal bones (M1-M5); notice that C2-M2 is slightly recessed proximal (arrow).

and three cuneometatarsal ligaments (these are thin ligaments) and the interosseous ligament C1-M2, the Lisfranc ligament. ${ }^{1,10}$ 


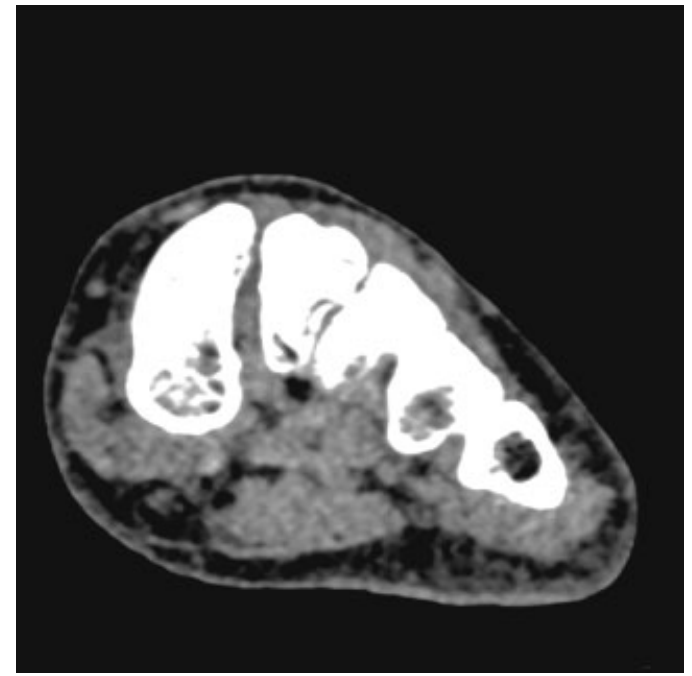

Fig. 3 Coronal foot computed tomography shows the shape of the midfoot arch and the wedge shape of the cuneiforms.

The Lisfranc ligament and the plantar Lisfranc ligament are distinct structures that can be differentiated on MRI. $6,7,10,11$ The Lisfranc ligament is the strongest and the largest of the Lisfranc joint ligaments (8-10 mm length $\times 5-6 \mathrm{~mm}$ thickness). It is an oblique striated ligament with one or two (and occasionally three) bundles coursing from the lateral wall of the medial cuneiform (adjacent to the intercuneiform ligament) to the medial base of the second metatarsal beyond the articular surface. ${ }^{2,7}$ Its plantar insertion is closely related to the interosseous C1-C2 ligament, plantar ligament, and peroneus longus. ${ }^{1,6,11}$ The Lisfranc ligament is better visualized on MR long axial

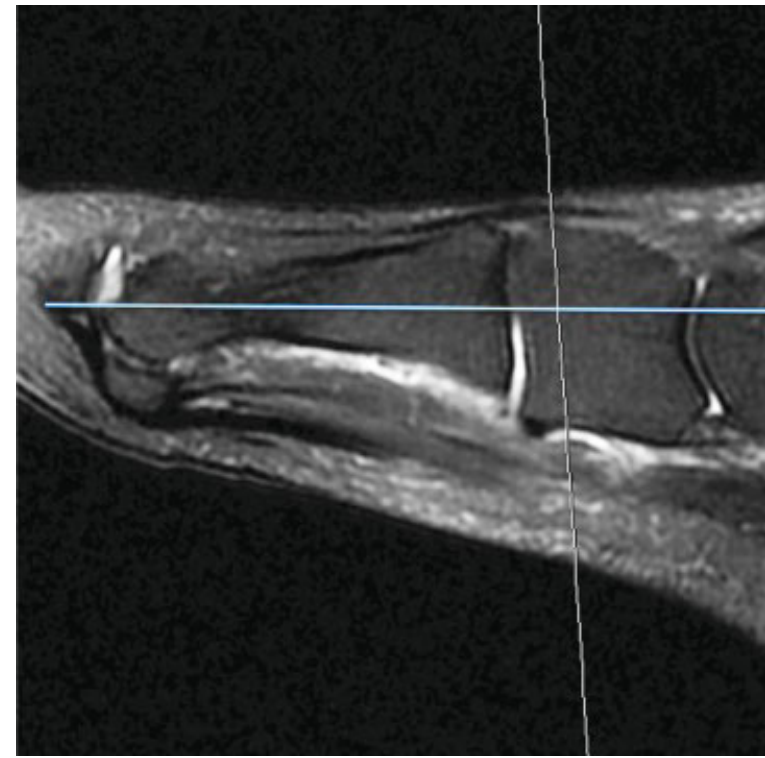

Fig. 5 MRI scan planning off the sagittal plane image. The axial plane follows the long axis of the metatarsals. The coronal plane is slightly oblique, perpendicular to $\mathrm{C} 1$.

planes and coronal planes The appearance on MR is variable (-Fig. 5). Castro et al described it as striated or homogeneous and low to intermediate SI. ${ }^{10}$

Plantar ligaments are stronger than the dorsal ligaments and course both longitudinally and transversely. Ligament strength decreases from medial to lateral. The first ligament is longitudinal and is the continuation of the naviculocuneiform ligament extending from $\mathrm{C} 1$ to M1. The second plantar ligament is the strongest and arises from

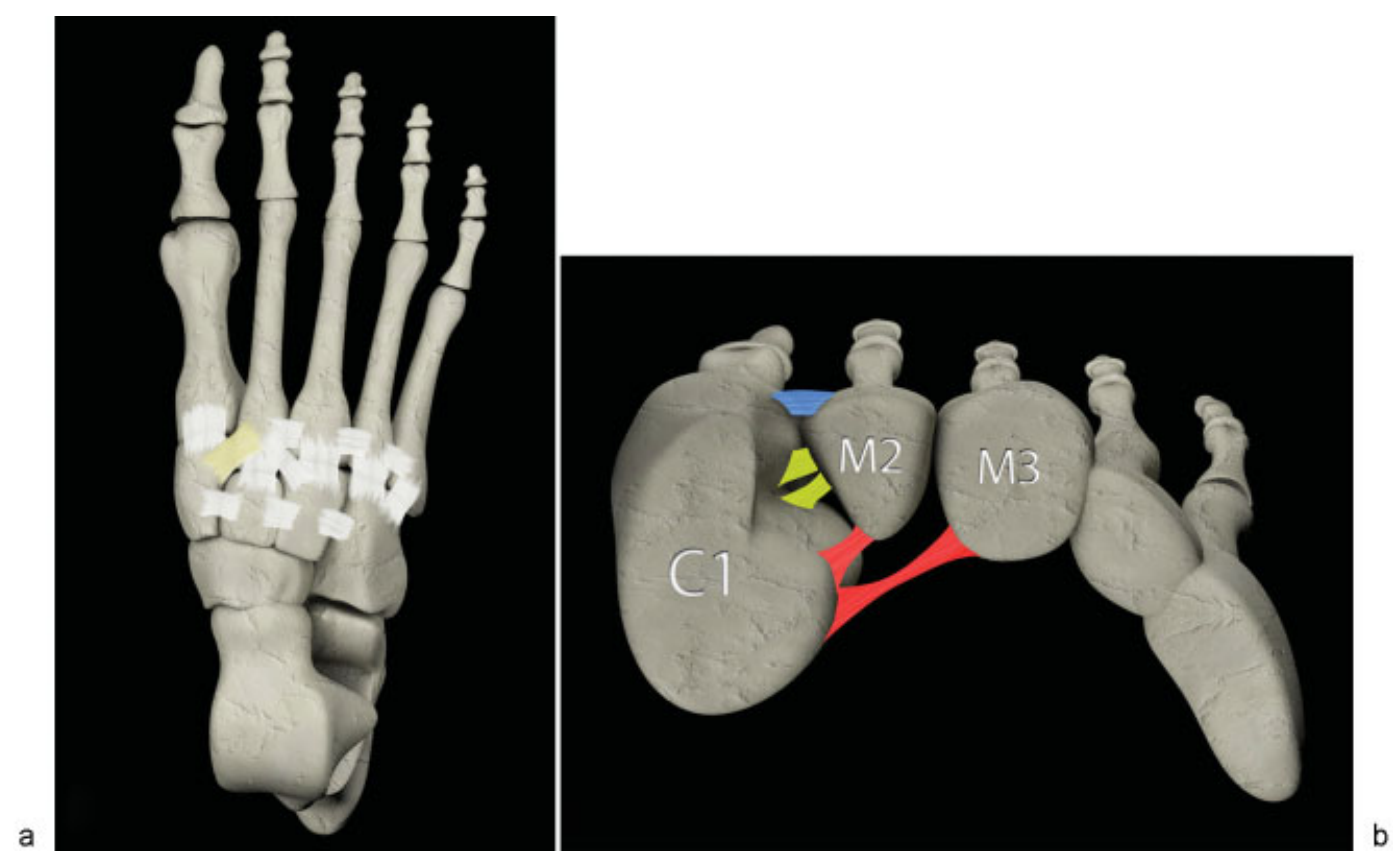

Fig. 4 Anatomical drawings of the midfoot ligaments. (a) Anteroposterior view of the dorsal ligaments. Note that there is no ligament between M1 and M2. (b) Coronal view: the ligaments between C1 and M2 are shown, the Lisfranc complex, the dorsal (blue), the two (yellow) bundles of the interosseous ligament (Lisfranc ligament), and the plantar (red) Lisfranc ligament with fascicles attached to M2 (deep) and M3 (superficial). 


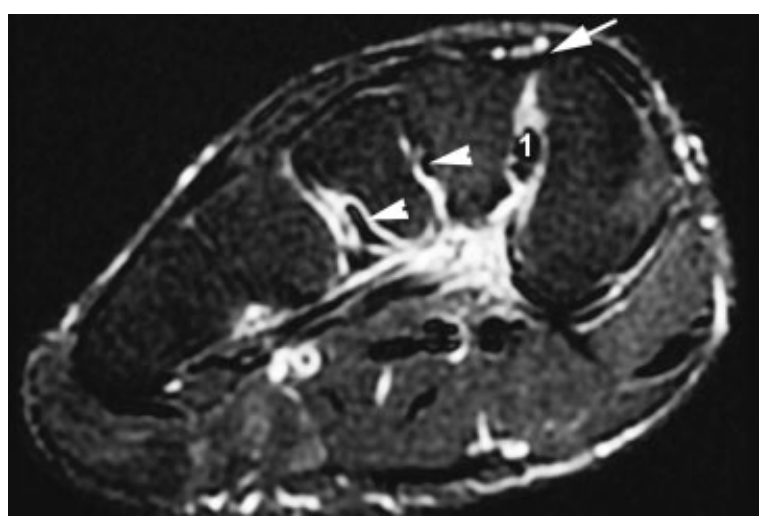

Fig. 6 Coronal plane fat-saturated fast spin-echo T2-weighted image nicely demonstrates the interosseous ligaments (arrowheads), dorsal ligament (white arrow), and Lisfranc ligament. ${ }^{1}$

C1 and attaches to M2-M3; although it may exhibit variable morphology, it usually has two bundles with the deeper attaching to M2 and the more superficial and thicker to M3. ${ }^{10,11}$ The second plantar ligament is difficult to visualize and better depicted in axial long-axis and coronal planes, appearing heterogeneous with low to intermediate signal intensity. ${ }^{11}$

The third plantar ligament connects the $\mathrm{C} 3$ to M3 and M4. The fourth and fifth plantar ligaments connect the cuboid to M4 and M5. The plantar intermetatarsal ligament is a band coursing between M1 and M3 without attachment to M2 (-Figs. 4-9). ${ }^{1}$

Additional support is provided by the soft tissues of the plantar foot including the tendons of the peroneus longus, anterior and posterior tibialis, long plantar ligament, plantar fascia, and intrinsic muscles. ${ }^{1}$

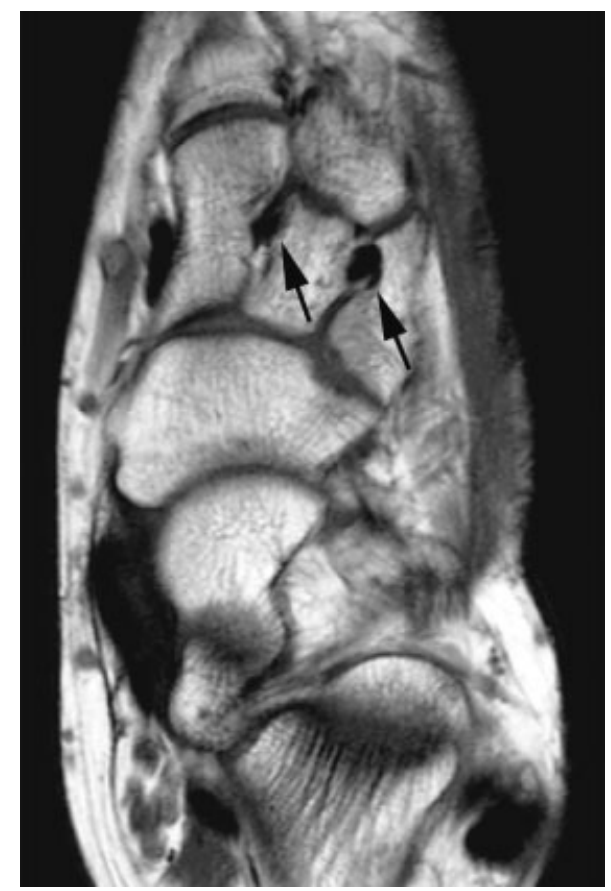

Fig. 7 Axial fast spin-echo T1-weighted image plane interosseous intertarsal ligaments (arrows).

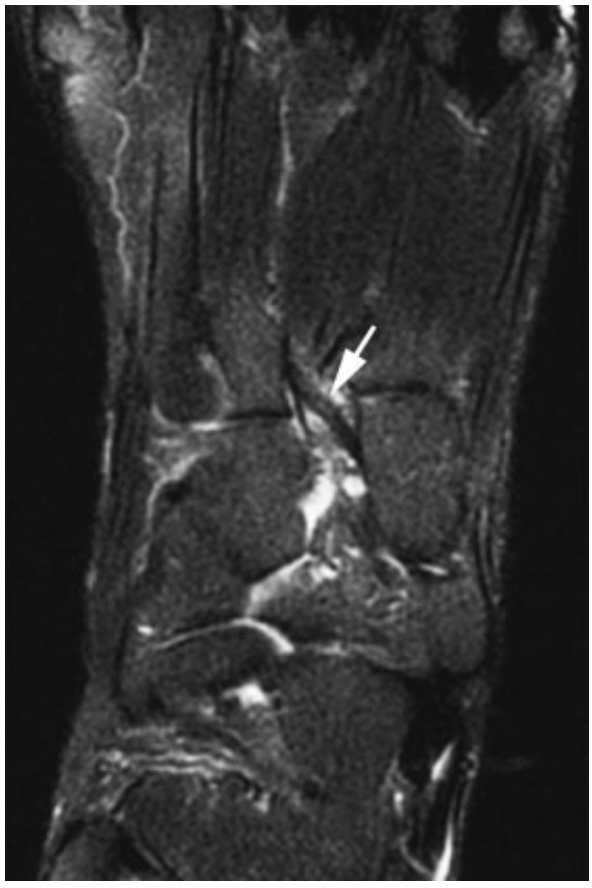

Fig. 8 Axial fat-saturated fast spin-echo T2 plane nicely demonstrates the plantar ligament of the superficial bundle inserting into M3.

\section{Biomechanics}

During the midstance phase of the walking gait, the midfoot facilitates forward progression of body weight on a stable foot, with a continuous transition from a flexible structure that dissipates the impact of the foot on contact with the floor to a rigid structure that allows propulsion during push-off. The functional columns of the foot allow for some motion and offset ( - Fig. 1). The medial and middle columns are more rigid than the more mobile lateral column, which tolerates up to a 2- to 3-mm offset. The medial column has little mobility, although slightly more

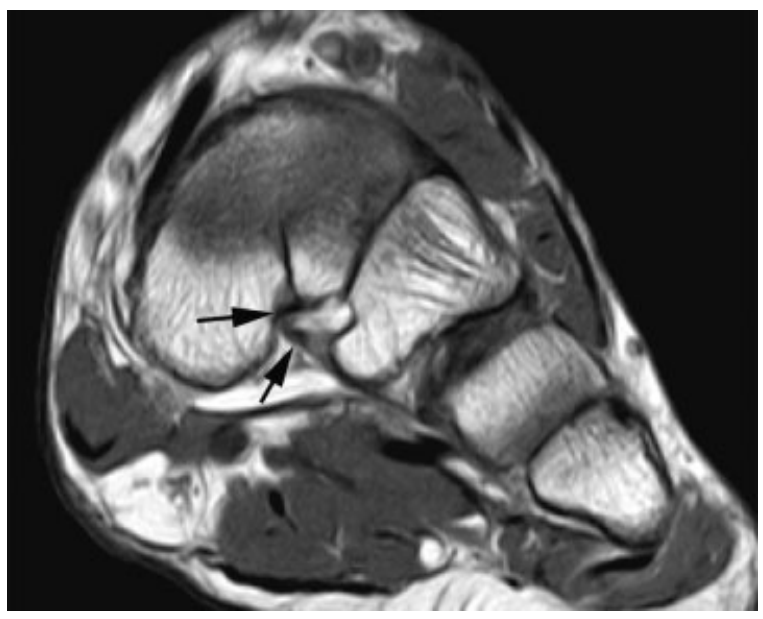

Fig. 9 Coronal fast spin-echo T1-weighted image shows the two bundles of the Lisfranc plantar ligament (arrows), the deep bundle inserting into $\mathrm{M} 2$ and the superficial inserting into $\mathrm{M} 3$. 
a
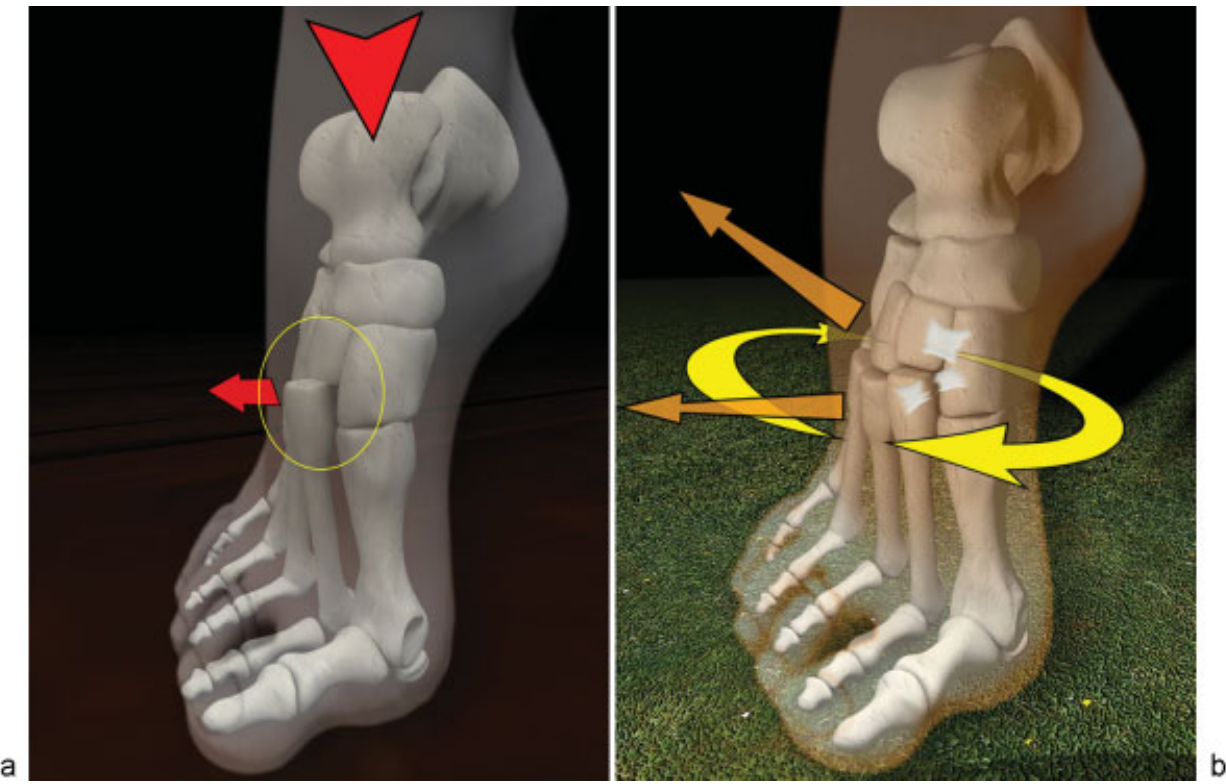

Fig. 10 Mechanism of injuries. (a) More frequent situation with the plantarflexed forefoot and a longitudinal force applied through the metatarsal bone. Alternatively, in (b) there is a rolling, twisting force over the midtarsal bones in the plantigrade, fixed forefoot.

than the middle column, which is almost fixed and does not tolerate instability or significant offset, $1 \mathrm{~mm}$. An offset $>1 \mathrm{~mm}$ between the middle and medial column may result in secondary OA.

The loss of the midfoot arch increases tensile stresses on the plantar ligaments as the foot is loading during push-off, thereby increasing the demands on muscular and ligamentous support. Moreover, the loss of the midfoot arch compromises its function as a rigid segment. ${ }^{12,13}$

Depending on the mechanism, injuries to the TMT joint can be direct or indirect. Direct injury is usually related to high-energy impact. Indirect injury occurs more frequently and can be associated with both high- and low-energy mechanisms.
Direct crushing injuries are often associated with significant blunt trauma to surrounding structures with associated soft tissue, vascular or neural injuries, and a possible risk for compartment syndrome. In high-energy trauma the displacement of the metatarsal bones depend on the vector of impact. This is often associated with multiple atypical tarsal bone fractures and surrounding soft tissue injuries. ${ }^{1-3,14}$

Indirect mechanisms can be due to high- or low-energy trauma. High-energy indirect mechanism is usually related to motor vehicle accidents or falls from a height, whereas lowenergy trauma typically occurs during sports. ${ }^{3,14}$ Low-energy injuries may be undiagnosed.

This sports injury most commonly occurs with excess loading when the foot is fixed in the plantar flexion and
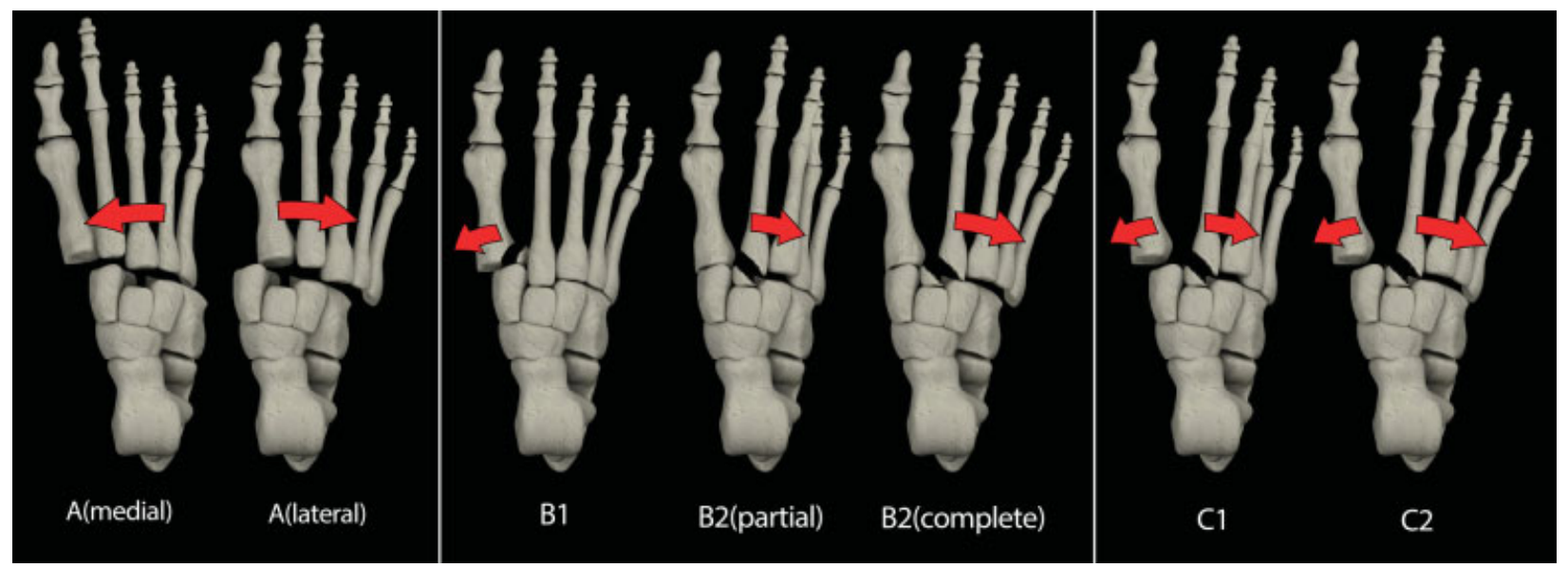

Fig. 11 Quénu and Küss classification modified by Myerson. Three categories: (a) all tarsometatarsal joints are disrupted with total incongruity lateral or medial, (b) partial incongruity, B1: medial displacement of the M1, and B2: lateral displacement of the lesser MT, and (c) divergent displacement partial or complete. 

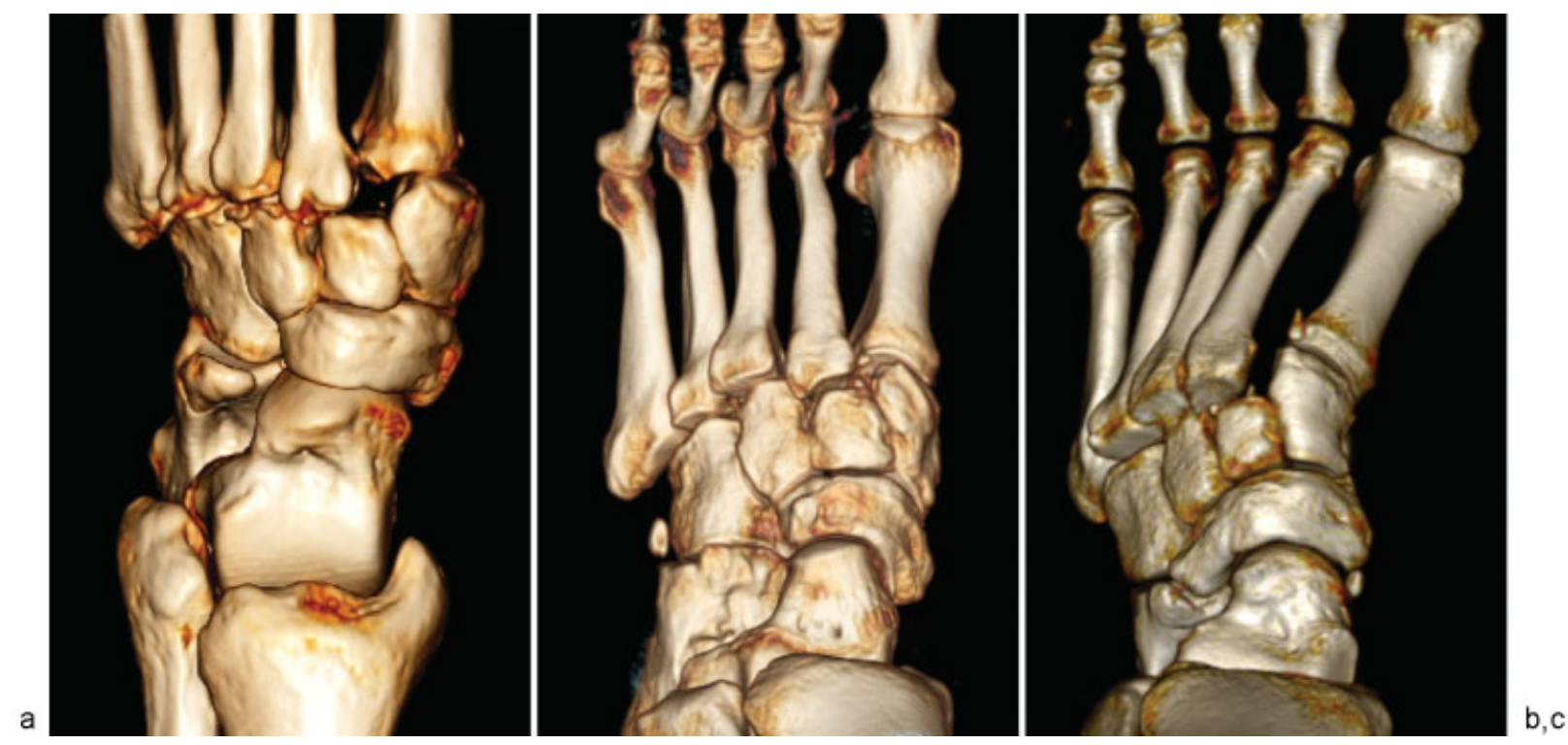

Fig. 12 Different examples of fractures dislocation on multidetector computed tomography with volume rendering reconstructions. (a) Type A with complete disruption and lateral and dorsal displacement. (b) Type B lateral partial incongruity of the lesser metatarsal (MT) with lateral and dorsal displacement of the lesser metatarsal bones. (c) Type $\mathrm{C}$ with divergent complete displacement. $\mathrm{M} 1$ is displaced medially, whereas the lesser MT is displaced laterally.

equinus position, primarily in American football, but ballet dancers and gymnasts are also at risk. A less frequent mechanism occurs when the forefoot is adducted, the hindfoot is fixed, and the weight of the body rotates around the TMT joint (-Fig. 10), as in falling from a horse with the foot fixed on the stirrup or rolling the foot when stepping off a step, curb, or jumping, resulting in dorsal displacement of the MT., ${ }^{1,3}$ Cavus deformation causes disruption of the dorsal ligaments, and a superimposed twisting moment leads to failure of the interosseous and plantar ligaments with resultant midfoot instability. ${ }^{14}$ Ligamentous injuries usually present in consistent patterns related to the relative strength of the structures. The dorsal ligaments tear first, followed by the plantar and finally the Lisfranc ligament. Although low-energy Lisfranc injuries comprise $0.2 \%$ of all fractures, they are important due to their predilection among young athletes and active workers, and because 20 to $35 \%$ are initially overlooked. Delayed diagnosis may predispose to instability and secondary OA. ${ }^{1,3}$

\section{Classification}

Classification systems differ depending on the mechanism of high-energy or low-energy impact lesions. High-energy impact lesions or crushing injuries result in fracture dislocations, whereas low-energy indirect forces result in Lisfranc injuries and sprains. ${ }^{9}$

Quénu and Küss in 1909 classified Lisfranc fractures, or fracture dislocation according to the direction of the MT
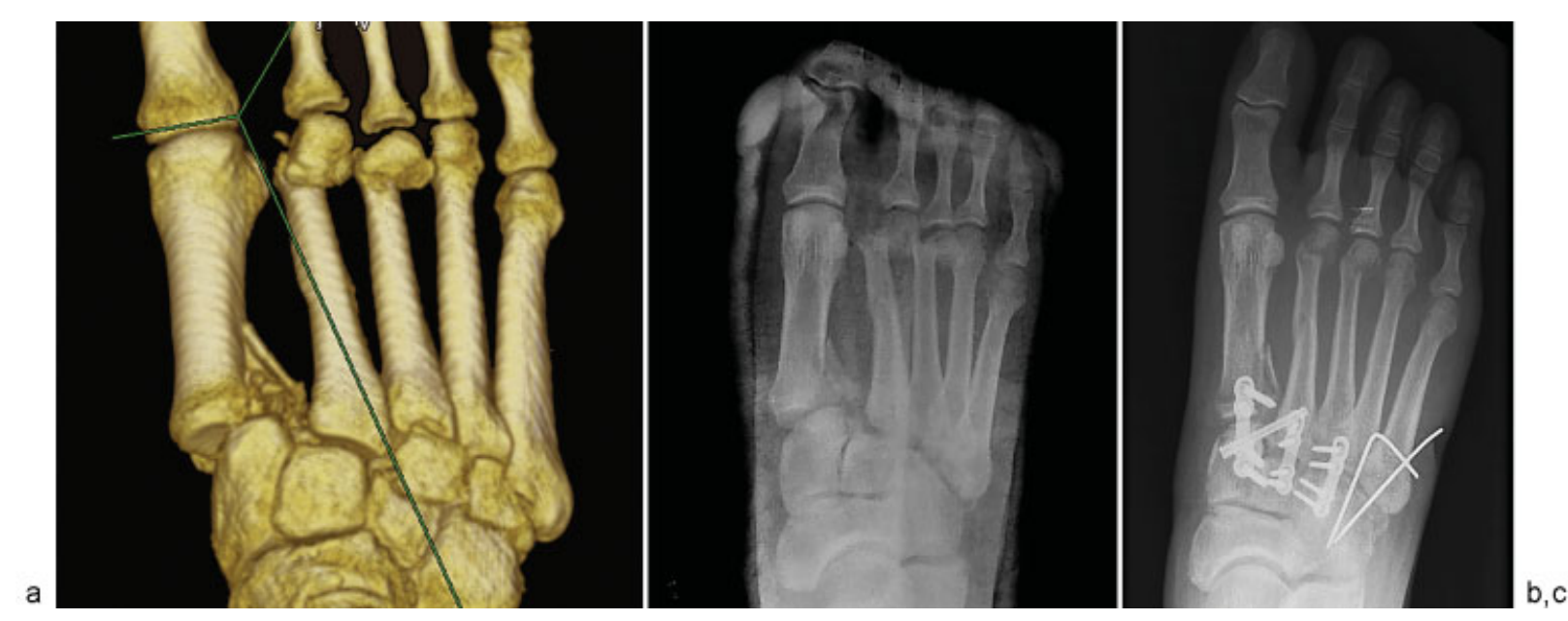

Fig. 13 Divergent complete fracture dislocation. (a) Multidetector computed tomography with volume rendering reconstruction. (b, c) Comparison plain radiographs before and after surgical reduction and fixation with screws and wires (courtesy of Dr. Solano). 


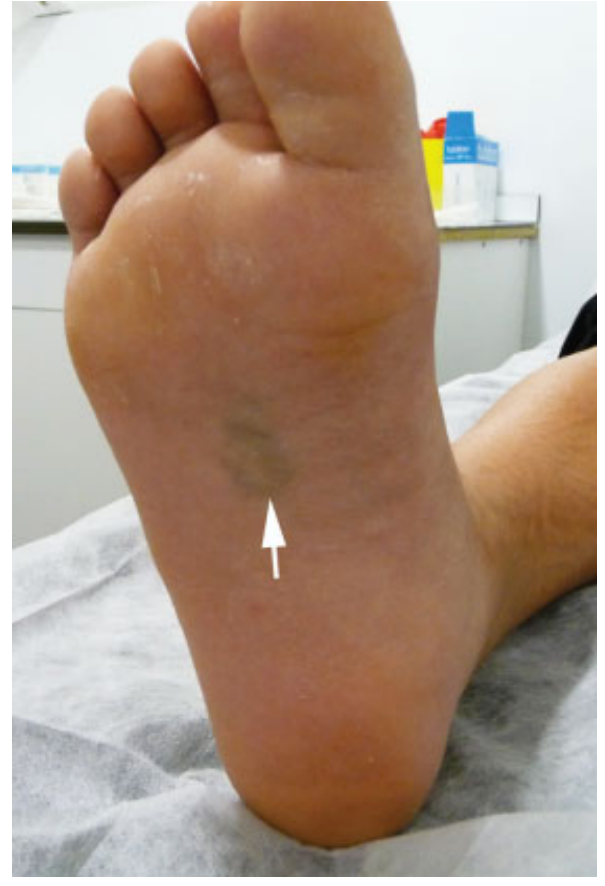

Fig. 14 The plantar ecchymosis sign is a classic clinical sign of Lisfranc injury. displacement as "homolateral" (all five MTs are displaced in the same direction), "isolated" (displacement of only one or two MTs), and "divergent" (metatarsals are displaced in different directions in the sagittal and coronal planes, M1 is displaced medially, and M2-M5 are displaced laterally). Homolateral is the most common. ${ }^{8,15}$ The Myerson classification is based on the columnar structure of the feet and the most commonly used. ${ }^{16}$ The Myerson classification divides fracture dislocations into three big categories: A (all the TMT joints are disrupted with total incongruity lateral or dorsoplantar), B (partial incongruity, B1, medial displacement of the $\mathrm{M} 1$, and $\mathrm{B} 2$, lateral displacement of the lesser MT), and C (divergent displacement is partial or complete) (-Figs. 11 and 12). Posttraumatic arthritis is more frequent at the middle cuneiform-second MT base where incongruity is less well tolerated. ${ }^{1,3,9,15-17}$ Because of the relatively more common delay in the diagnosis, type B tends toward a worse prognosis. ${ }^{8}$ Multidetector computed tomography (MDCT) provides an exquisite map of fracture fragment displacement for treatment planning (-Fig. 13).

Fracture dislocation classifications systems are not useful for low-energy Lisfranc injuries without fractures. These
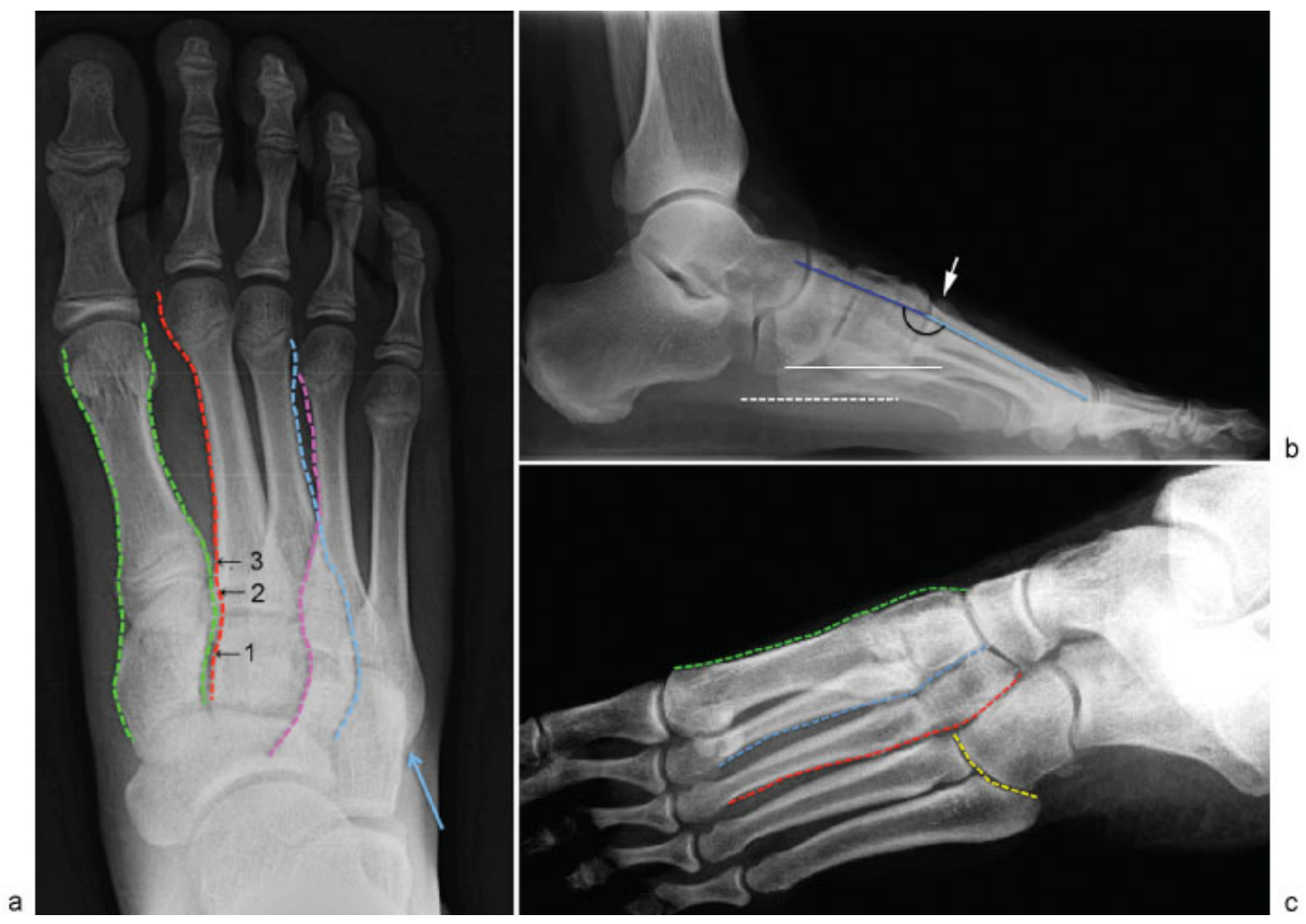

Fig. 15 Different film projections of the midfoot normal radiologic anatomy. (a) Anteroposterior foot plain film showing the normal metatarsal lines: first metatarsal line lateral and medial to M1 (green); second line medial border of M2 should align with the medial border of C2 (red). Third line lateral border of M3 aligns with the lateral border of $\mathrm{C} 3$ (blue); the medial border of $\mathrm{M} 4$ aligns with the lateral border of the cuboid bone (pink). The base of M5 should not be displaced (blue arrow). Intertarsal space should be symmetrical C1-C2, C1-M2, and M1-M2. (b) Lateral weightbearing film. Note that there is no step-off at the dorsal margin of the tarsometatarsal joint (white arrow); talometatarsal angles should be $<10$ degrees. The plantar margin of $\mathrm{C} 1$ (white line) should lie dorsal to the plantar margin of the base of M5. (c) Oblique normal film is optimal for assessing M3, M4 alignment with C3, and the cuboid bone. Medial border of M1 and medial border of $\mathrm{C} 1$ should be smooth and continuous (green line); the lateral margins of C2-M2 and C3-M3 (blue and red lines) should also be aligned. The fourth and fifth metatarsal articulate with the cuboid bone (yellow line). 


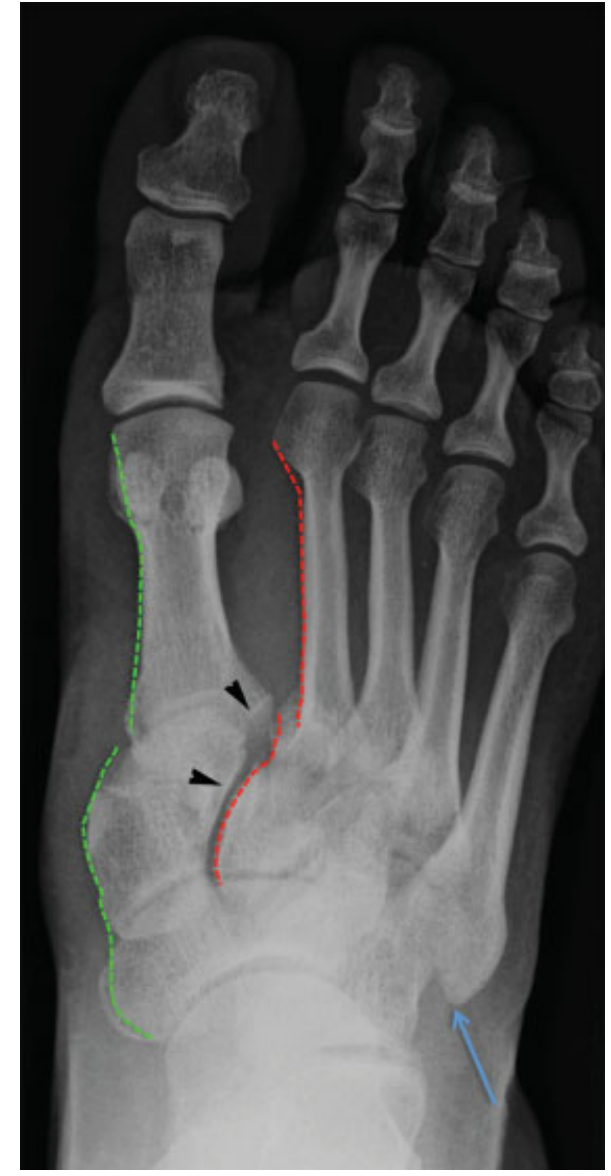

Fig. 16 Lisfranc fracture dislocation. (a) Disruption of the first column line (green line). (b) Disruption of the middle column line, C2-M2 (red line). (c) Widening of the intertarsal space between $\mathrm{C} 1$ and $\mathrm{C} 2$ and intermetatarsal space M1-M2 (black arrows). (d) Lateral displacement of the base of M5 (blue arrow).

low-energy Lisfranc injuries or midfoot sprains can be easily undiagnosed because of their subtle clinical and radiologic findings. Curtis et al classified midfoot sprains into first- and second-degree injuries (partial tears without instability on clinical or fluoroscopic examination), and third-degree injuries (complete rupture with diastasis on radiographs). ${ }^{17}$ Nunley and Vertullo ${ }^{18}$ combined clinical, radiographic, and bone scintigraphy findings into a classification system with management implications. They described three stages. Stage I is a low-grade sprain of the Lisfranc ligament complex in which the patient complains of pain in the Lisfranc joint, the plain films are normal (no C1-M2 diastasis and normal midfoot arch), but bone scans show uptake. These injuries are treated conservatively. Stage II is due to elongation, partial or complete tear of the Lisfranc ligament with an intact plantar capsular ligament; plain films show $<5-\mathrm{mm}$ diastasis at $\mathrm{C} 1-\mathrm{M} 2$ and M1-M2. Stage III implies disruption of the dorsal, Lisfranc, and plantar ligament, with $>5-\mathrm{mm}$ M1-M2 diastasis on an anteroposterior (AP) weightbearing radiograph and loss of arch height on lateral standing radiography. If displaced injuries are present, other associated fractures should be

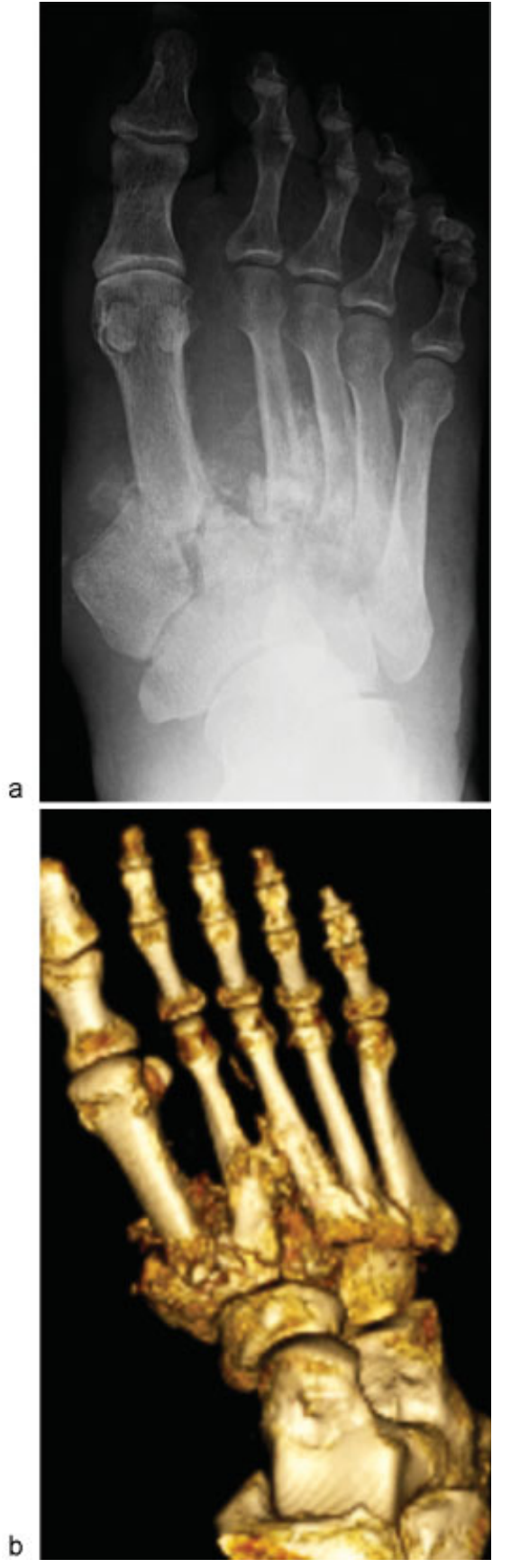

Fig. 17 Complete fracture dislocation type A with lateral displacement, anteroposterior $\mathrm{x}$-ray (a) and the corresponding multidetector computed tomography with volume rendering (b).

classified using Myerson's system. Stage III requires surgical treatment. Treatment of stage II is debatable, although the tendency is toward surgery. ${ }^{1-3,9,18}$

\section{Clinical Symptoms}

The diagnosis of Lisfranc fracture dislocation is straightforward when the patient presents with deformity, swelling, widening of the midfoot, and a flat forefoot deformity. Occasionally in the setting of multitrauma, especially automobile injuries or falls from a height, Lisfranc fractures can 

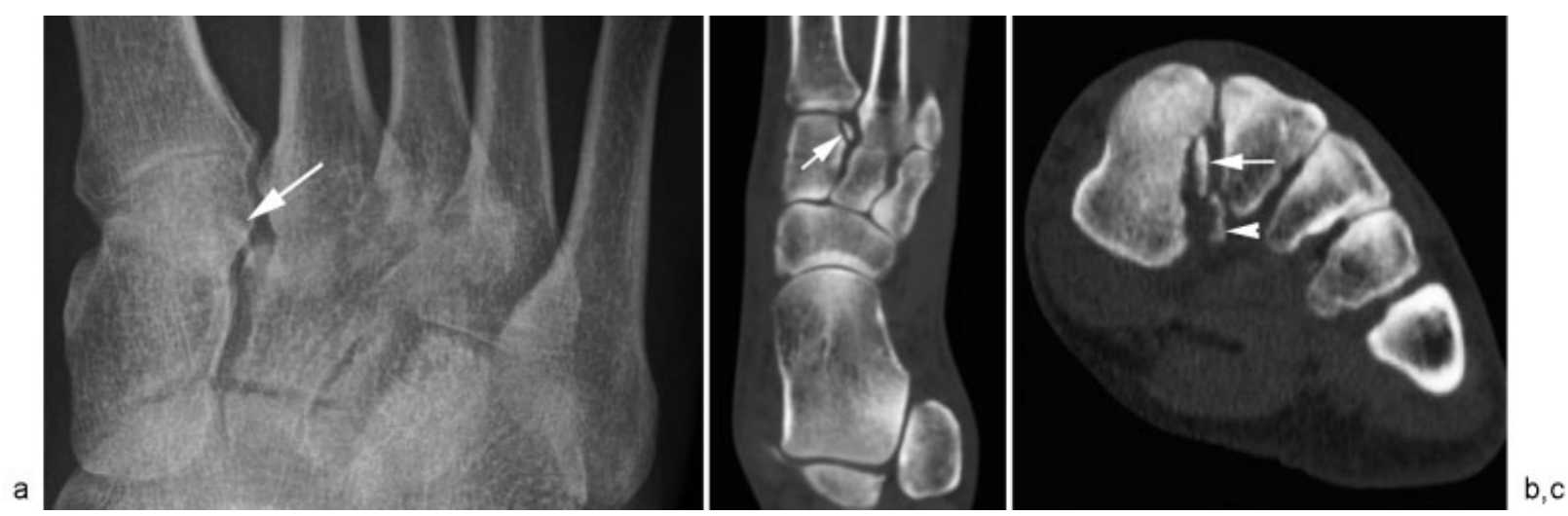

Fig. 18 (a) Fleck sign, small fragment avulsion, can be depicted on the anteroposterior film. (b) However, it is easier to confirm it with multidetector computed tomography, transverse multiplanar reformation (MPR) reconstruction. (c) Coronal CT MPR reconstruction nicely demonstrates the two fragments avulsion corresponding to the Lisfranc ligament avulsion and the plantar ligament avulsion (arrows).

reduce spontaneously. In those cases where there is no gross deformity, clinical diagnosis is more difficult and midfoot instability should be ruled out.

Compartment syndrome is a potential complication, to be considered when severe pain and swelling is present. When clinically suspected, pressure measurements should be performed. Although infrequent, injuries to the dorsal pedis artery and deep peroneal nerve injury should be excluded. ${ }^{3}$ Although Lisfranc sprains are much more difficult to diagnose by imaging than fracture or fracture dislocation, subtle clinical signs include midfoot swelling, inability to bear weight, and especially plantar arch hematoma (the plantar ecchymosis sign $)^{3,19}$ (- Fig. 14). The piano key test assesses TMT joint pain: the hind- and midfoot are fixed and plantar force is applied to the metatarsal heads. ${ }^{20}$

\section{Imaging}

\section{Plain Film}

Radiographic evaluation of the TMT joint is difficult due to osseous overlap. After midtarsal trauma, initial films are non-weightbearing AP, lateral and internal oblique views (30 degrees). It is important to keep in mind that subtle diastasis can be missed in up to $50 \%$ of cases on non-weightbearing radiographs. If there is a strong clinical suspicion, ${ }^{8,21}$ weightbearing films of both feet are required for comparison with the uninjured contralateral foot to rule out subtle diastasis or small displaced injuries (- Fig. 15). ${ }^{1,22}$ The pronation abduction stress view has been advocated to rule out instability of the first ray, but anesthesia is frequently needed to control pain. With the advent of new imaging techniques, however, stress views are rarely necessary. ${ }^{14}$ The distance between the cuneiform and the metatarsal bones can be variable; therefore, it is important to compare with the uninjured contralateral foot; asymmetry $>1$ to $2 \mathrm{~mm}$ should raise suspicion of instability, ligament injuries, or occult fractures. ${ }^{1,8,21,22}$

Alignment should be assessed in the AP view. The lateral margin of the first TMT and medial margins of the second and third TMT should each align almost perfectly, and the medial border of $\mathrm{C} 2$ should align with the medial border of the base of the second MT. In the oblique view the lateral margins of C2M2 and C3-M3 should align, and the fourth and fifth MT should articulate with the cuboid bone. Weightbearing lateral films are used to assess the medial plantar arch and to detect possible dorsal subluxation of the metatarsal bases (-Fig. 15) ${ }^{8,14}$

Diagnosis of Lisfranc fractures and Lisfranc injuries is challenging. Subtle fractures of the metatarsal bones and midfoot malalignment must be evaluated. Disruption of the continuous tarsal metatarsal lines of the first and second column and displacement of the base of M5 or dorsal displacement of the metatarsal bones are reliable signs of fracture dislocation (-Fig. 16). Diastasis $\geq 2 \mathrm{~mm}$ between $\mathrm{M} 1$ and M2 indicates instability. ${ }^{8}$ If complex fractures are suspected on plain film, MDCT should be performed for preoperative evaluation (-Fig. 17). Small fractures often occur at the medial base of M2 or of the plantar lateral

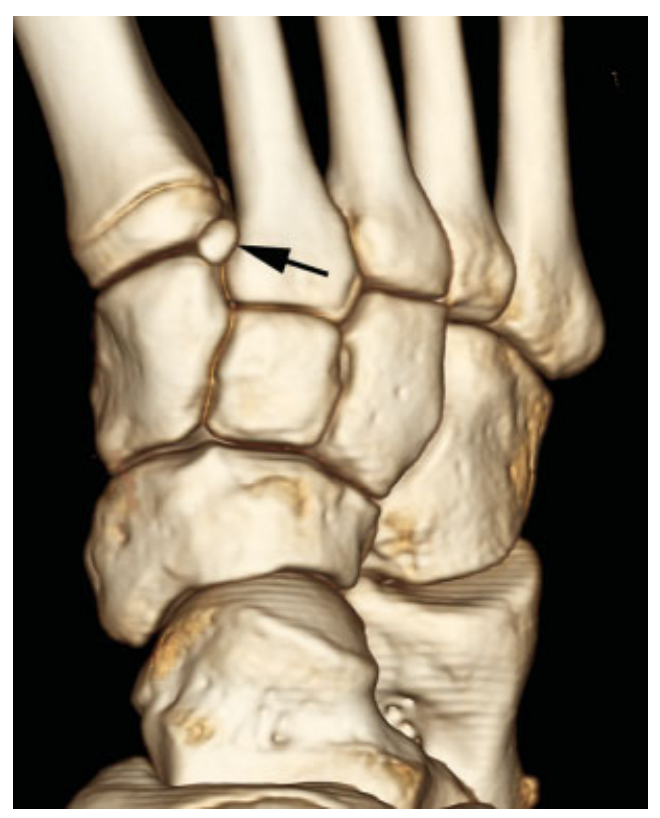

Fig. 19 This normal variant, os intermetatarseum, should not be mistaken for an avulsion fracture. 


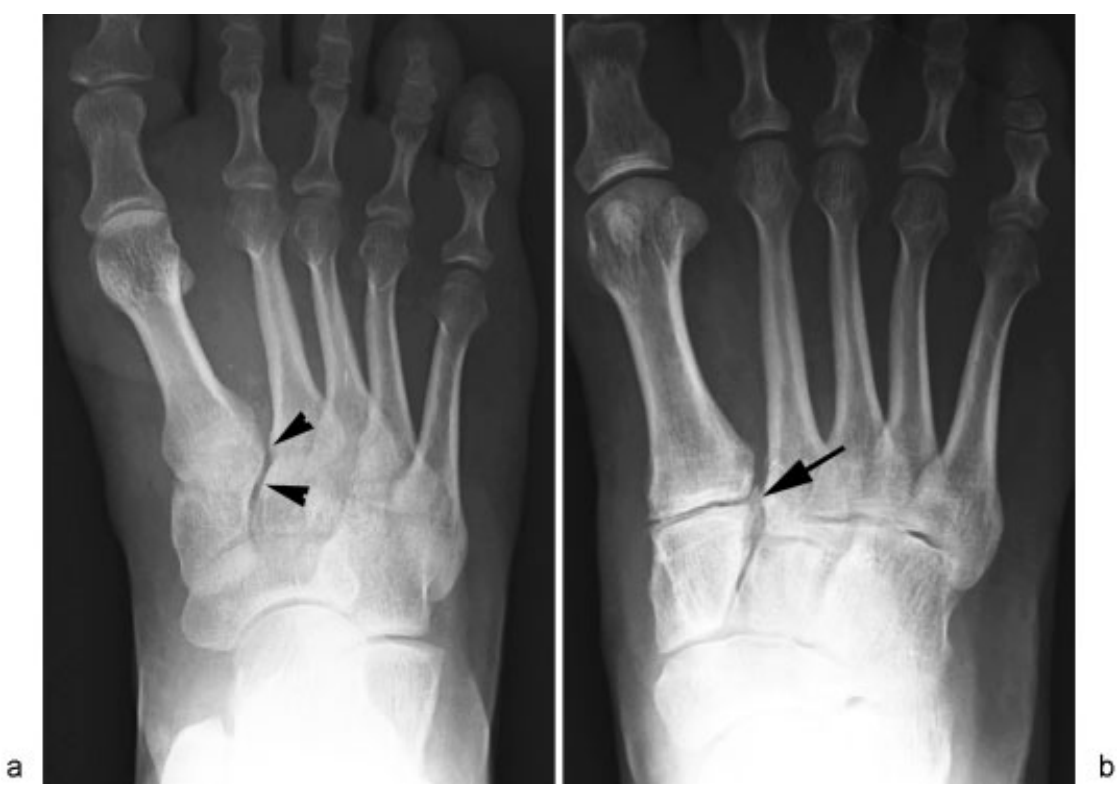

Fig. 20 (a) Lisfranc injury: anteroposterior foot film shows increased distance between C1 and C2 and M1 and M2. (b) Corresponding weightbearing film demonstrates a small fleck fracture (arrow) between C1 and M2.

base of $\mathrm{C} 1$. The fleck sign is a subtle cortical avulsion fracture at the attachment of the Lisfranc ligament (-Fig. 18). We must not confuse a fleck with a normal variant small ossicle (os intermetatarseum), which is located more distally and appears round to ovoid and smoothly corticated (-Fig. 19). Weightbearing films are useful to better depict small fractures ( - Fig. 20), visualize diastasis between the foot columns (especially between the first and second), and compare with

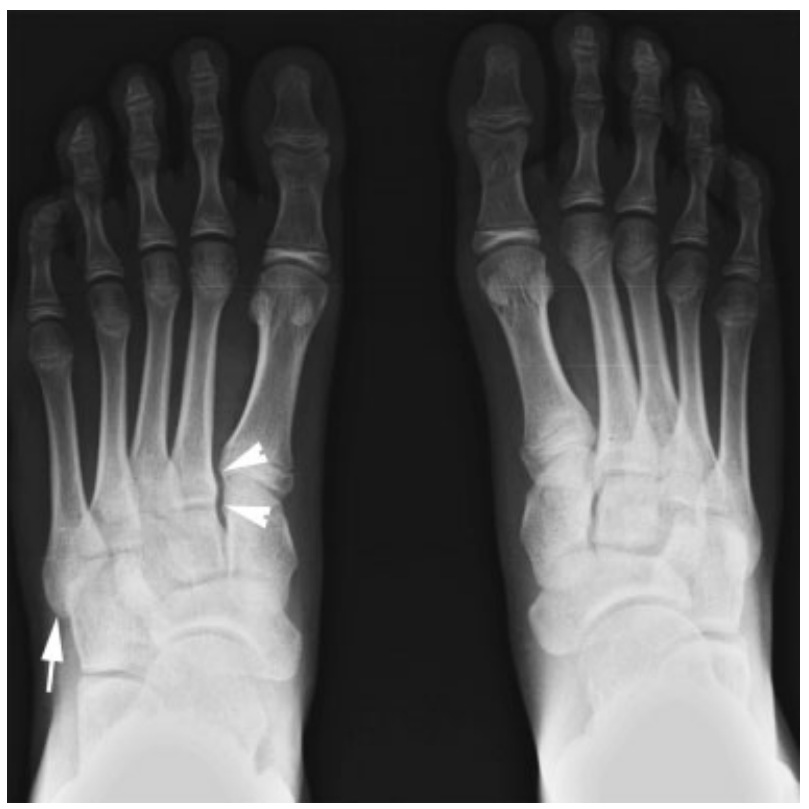

Fig. 21 Weightbearing anteroposterior films illustrate that subtle differences are easier to detect comparing the injured foot with the uninjured contralateral foot. Right foot is normal. In the injured left foot there is a relative increase in the distance between $C 1$ and $C 2$ and M1 and M2 (arrowheads), and prominent base of M5 (white arrow). No fractures were seen. the contralateral uninjured foot ( - Fig. 21). Widening $>2$ $\mathrm{mm}$ at $\mathrm{C} 1-\mathrm{M} 2$ or $>1 \mathrm{~mm}$ at $\mathrm{M} 1-\mathrm{M} 2$ or $\mathrm{C} 1-\mathrm{C} 2$ are the most important radiographic signs. Lateral films are used to assess dorsal displacement of the MT bases of and to measure the distance between the plantar margin of C1 and M5 (which is abnormal if $<1.5 \mathrm{~mm}$ ) or asymmetric as compared with the contralateral foot.

Because nuclear bone scintigraphy is sensitive but lacks specificity, it is not frequently used. Some surgeons use it, however, in the setting of secondary OA to determine which joints require fusion. ${ }^{8,21}$

CT is an important preoperative tool for the evaluation of fracture pattern and surgical planning in patients after highenergy trauma when complex fractures are suspected ${ }^{3,8}$ (-Figs. 12,13, and 17). CT permits detection of 50\% more metatarsal and tarsal fractures compared with radiographs. $\mathrm{CT}$, especially volume rendering, is also more accurate for the evaluation of osseous malalignment, helping surgical planning ${ }^{1,10,23}$ (-Figs. 12, 13, 17, and 22). ${ }^{1,10}$

Unlike CT, MR allows direct visualization of soft tissue structures. Imaging should be performed in the sagittal plane, long axial plane (following the metatarsal bone axis), and oblique coronal plane (perpendicular to $\mathrm{C} 1$ ) to visualize the ligament complex (-Fig. 5). T1-weighted and fluid-sensitive sequences (be it T2-weighted or proton-density fat saturation or short tau inversion recovery) are recommended. Small surface coils might be useful to improve signal. Three-dimensional fast spin-echo images can provide thin slices for multiplanar reformation reconstructions, although technical issues remain before volumetric imaging of the midfoot is extended to routine clinical practice. ${ }^{11}$ Although occult fractures can be detected by MR and may reveal subtle ligament injuries, it does not change the management of complex bone fractures (-Fig. 23). MR is primarily important for the diagnosis and management of 


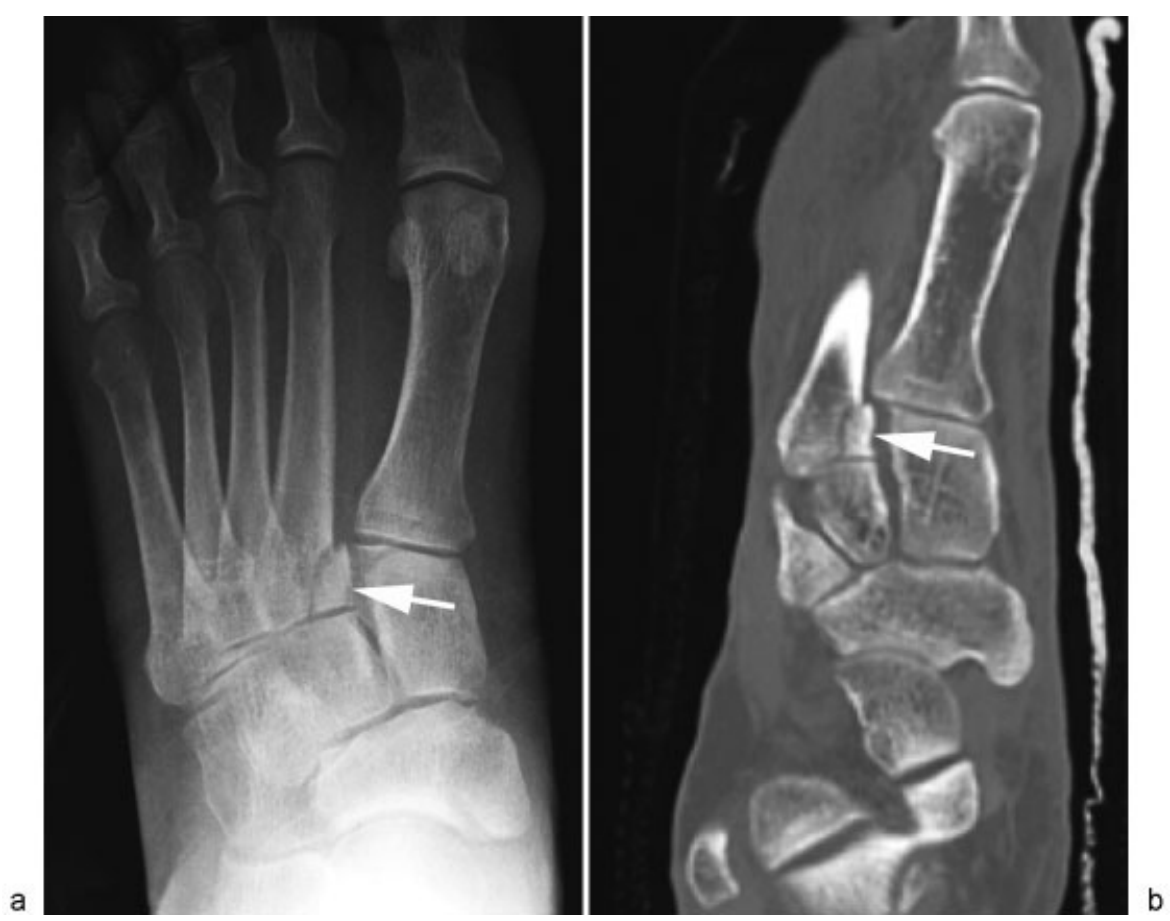

Fig. 22 (a) Plain film and (b) computed tomography with concordant demonstration of small avulsion at the base of M2 (arrow).

low-energy Lisfranc injuries, and it is recommended when there is minimal diastasis between the first column and the second cuneiform, or a high clinical suspicion of Lisfranc injury in the context of normal radiographs. MR depicts the distinct dorsal and plantar fascicles of the Lisfranc ligament, allowing diagnosis of partial or complete tears ( - Figs. 24-27). Usually complete tears of the ligament are associated with diastasis $\mathrm{C} 1-\mathrm{C} 2, \mathrm{C} 1-\mathrm{M} 2>2 \mathrm{~mm}$. The management of partial tears is still to be determined. A small Lisfranc ligament tear may remain occult, and occasionally the ligament can be stretched (elongated) but not torn. The presence of fluid surrounding the ligament should raise suspicion of a Lisfranc injury, especially if there is associated bone marrow edema, contusions, or fractures at the ligament insertion sites. In the chronic phase, diagnosis is more difficult because there may be irregularity and signal heterogeneity of the injured ligament due to the fibrotic healing response $e^{1,11}$ (-Fig. 28).

MR is also useful for assessment of commonly associated injuries, to tendons (anterior tibialis) or vital structures, such as the deep peroneal nerve.

\section{Clinical Management}

\section{Nonoperative Treatment}

Stable nondisplaced fractures, stage I, can be managed conservatively in a non-weightbearing cast for 4 to 6 weeks, followed by a weightbearing special shoe. Refractory pain should prompt reimaging to rule out major injuries or instability.
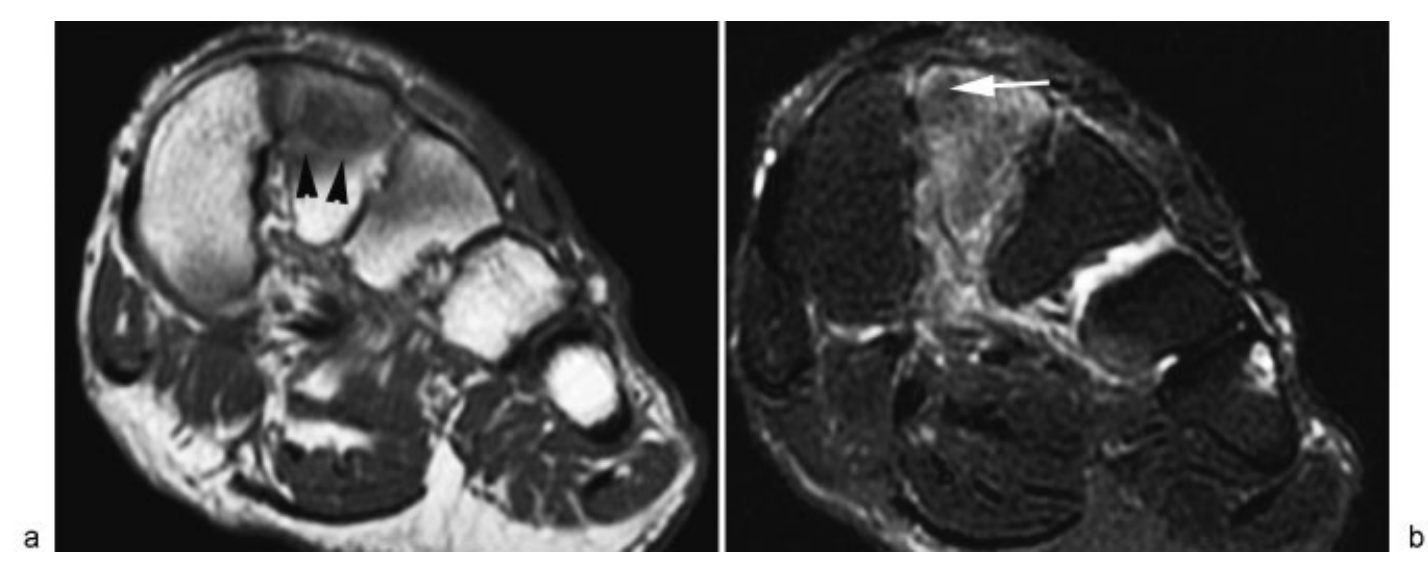

Fig. 23 Small fracture of C2. (a) Coronal fast spin-echo (FSE) T1-weighted image, and (b) the corresponding fat-saturated fast spin-echo (FSE) T2weighted image show bone marrow edema and small fracture line (arrowheads), and edema in the dorsal ligament (arrow). 


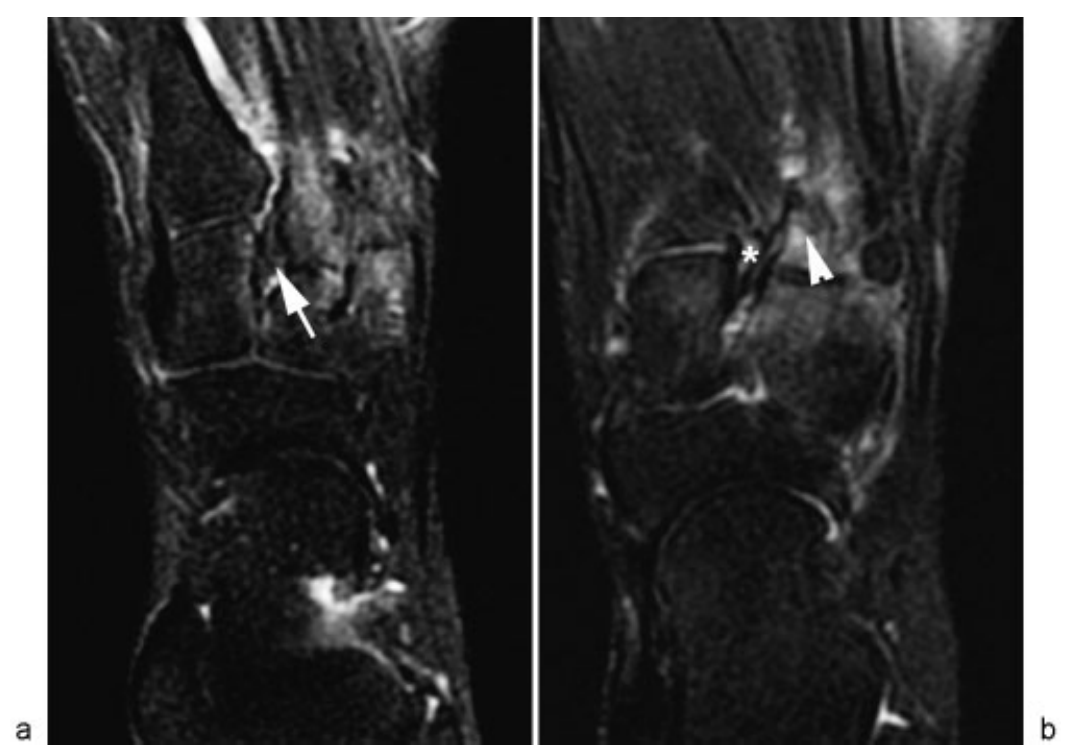

Fig. 24 Lisfranc subtle injury. (a, b) Consecutive transverse fat-saturated fast spin-echo T2-weighted images show elongation and soft tissue edema of the Lisfranc ligament (arrow) and of the plantar ligament in its superficial portion (asterisk) with bone marrow edema and small nondisplaced fracture on the base of M3 (arrowhead).

\section{Operative Treatment}

There is no consensus for the treatment of stage II. Most orthopedists recommend surgery despite minor displacement, whereas others favor conservative management after checking TMT joint stability. If conservative treatment is preferred, careful clinical surveillance for developing signs of instability is essential. Surgical treatment is indicated for stage III Lisfranc injuries and fracture dislocation. Better outcomes have been shown with early anatomical reduction and stable fixation. Surgery should be performed after swelling has decreased, ideally within 1 to 2 weeks of the initial injury. The goal of treatment is to achieve a painless, stable,

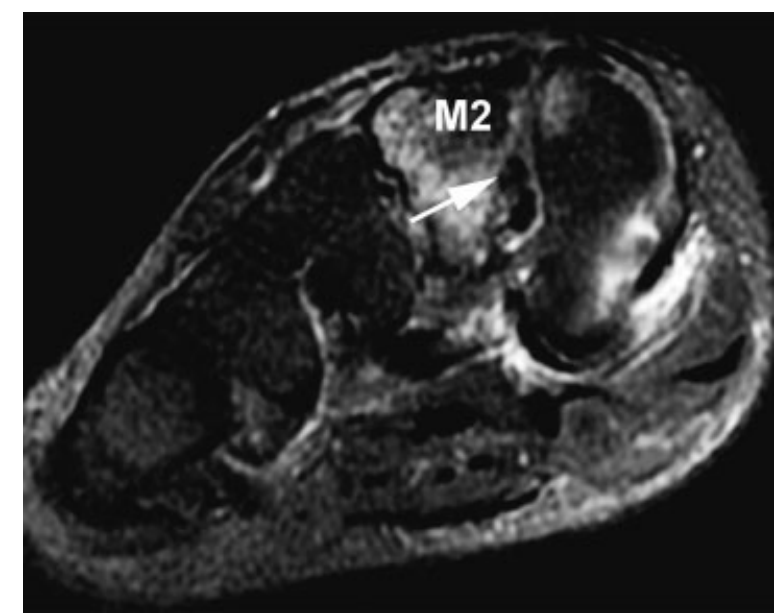

Fig. 25 Lisfranc subtle injury. (a, b) Consecutive coronal coronal fatsaturated T2-weighted images demonstrate the two fascicles of the Lisfranc ligament, with edema in the dorsal fascicle (arrow), whereas the plantar fascicle is normal. Note bone marrow edema without displaced fracture in M2. and plantigrade foot. Care should be taken in the anatomical reduction of the Lisfranc joint. Closed reduction and percutaneous Kirschner wire (K wire) fixation suffices in some cases. If closed anatomical reduction cannot be achieved, open reduction and internal fixation surgery with $\mathrm{K}$ wires or cortical screws should be performed ( - Figs. 28 and 29). ${ }^{10,13,22}$ When there are comminuted fragments, failure of fixation, or secondary $\mathrm{OA}$, arthrodesis is preferred (-Fig. 30). Medial and middle column fixation is typically performed. If reduction is achieved, lateral column fixation is usually unnecessary and is associated with improved outcomes ( - Fig. 31 $)^{8,13}$

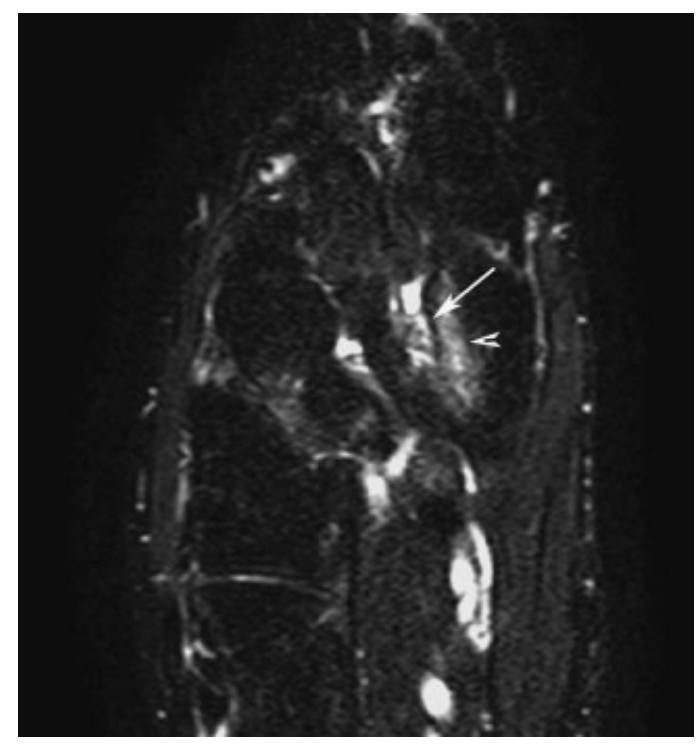

Fig. 26 Transverse fat-saturated fast spin-echo T2-weighted image demonstrates a partial rupture of the Lisfranc ligament (arrow) with edema within the fibers and in the soft tissue. 


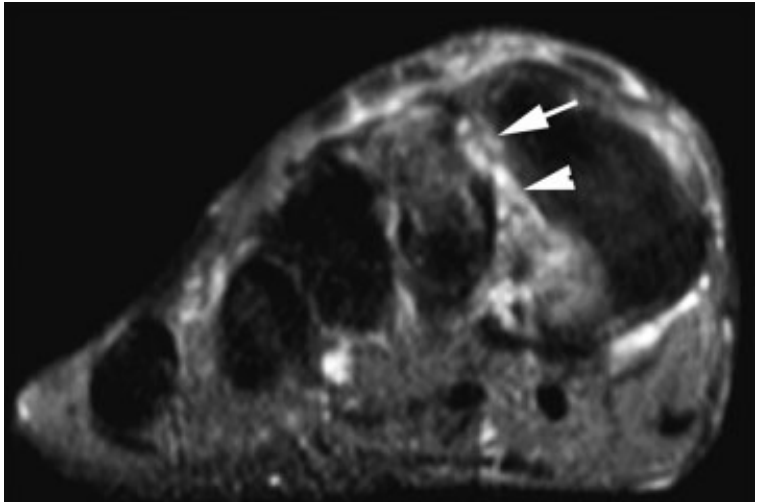

Fig. 27 Fat-saturated fast spin-echo T2-weighted image shows the rupture of both fascicles of the Lisfranc ligament (single arrow, dorsal fascicle; arrowhead, plantar fascicle).

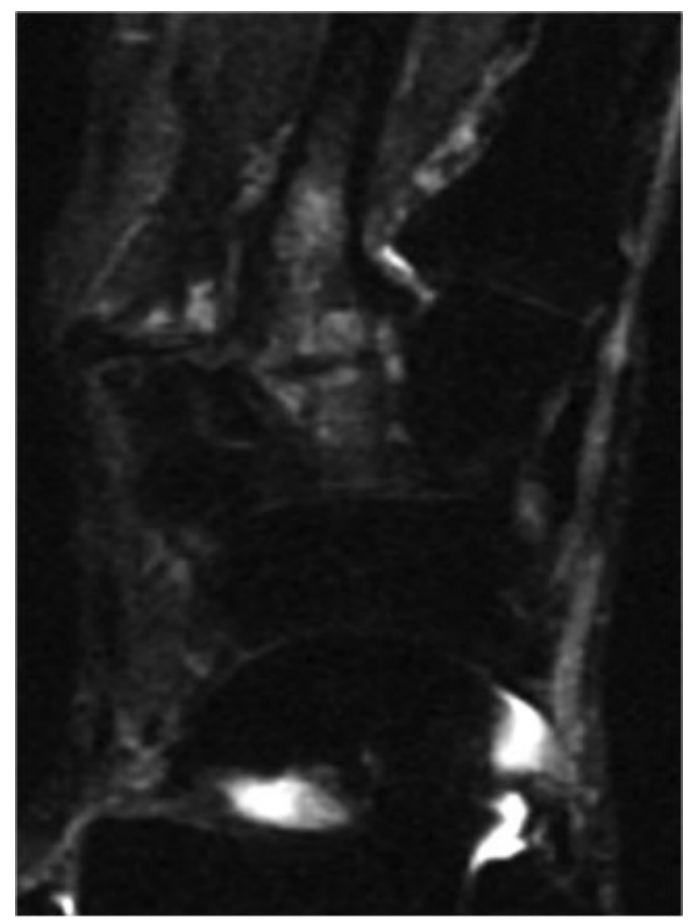

Fig. 28 Transverse fat-saturated fast spin-echo T2-weighted image of a chronic Lisfranc ligament rupture 3 years after trauma. The ligament is thickened with irregularity in the insertions on $\mathrm{C} 1$ and $\mathrm{M} 2$. Note the degenerative changes on $\mathrm{C} 2-\mathrm{M} 2, \mathrm{C} 3-\mathrm{M} 3$.

\section{Midfoot Osteoarthritis}

Midfoot OA is more common than previously thought and a challenging problem. Clinical symptoms include pain, swelling, difficulty standing and ambulating, and progressive deformity. $^{12}$

Posttraumatic OA is most common, followed by idiopathic and inflammatory etiologies. Posttraumatic OA origin usually occurs at $\sim 40$ years of age, whereas other nontraumatic etiologies typically present in an older population, at $\sim 60$ years. The etiology of nontraumatic midfoot $\mathrm{OA}$ is multifactorial, and risk factors include obesity, female

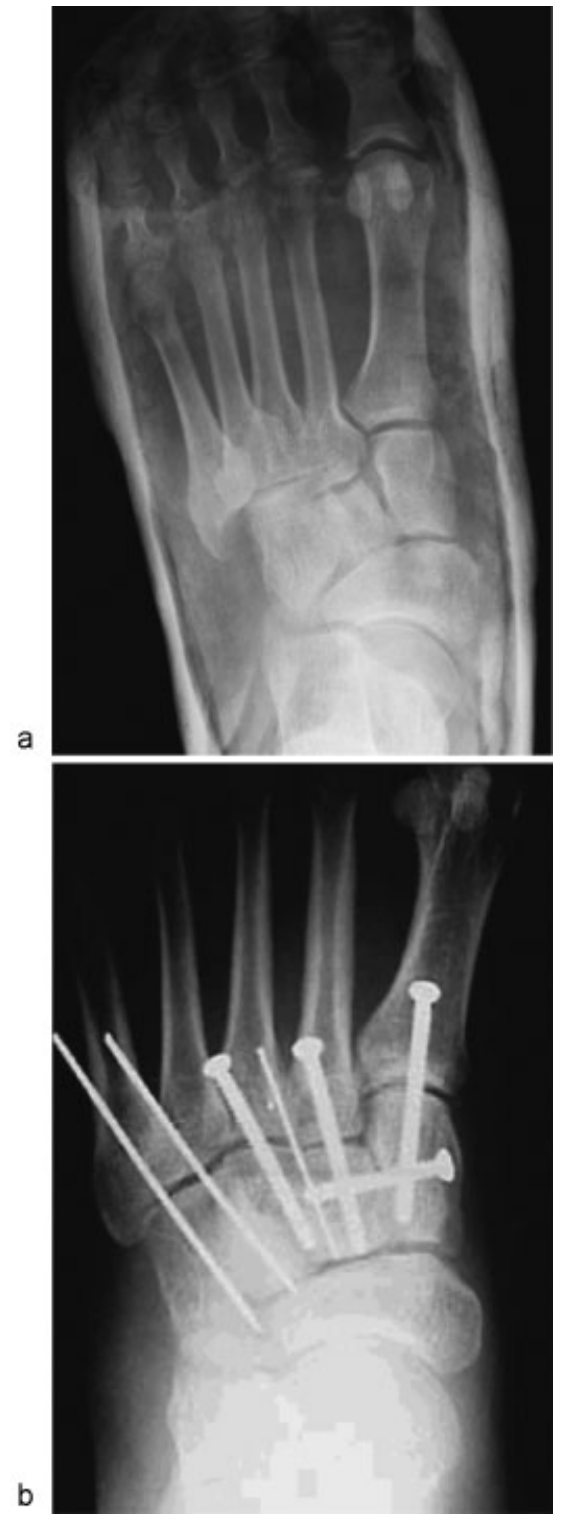

Fig. 29 Lisfranc fracture dislocation type A (a) with lateral displacement of the lesser metatarsal bones treated with wires and screws (open reduction and interval fixation) (b).

gender, equinus foot morphology, and development elongation of M2.

Primary OA preferentially affects the second and third MT middle column, whereas posttraumatic OA tends to affect the first, second, and fourth TMTjoints ${ }^{6,12,20}$ (-Figs. 28 and 32 ).

Conventional radiographs and CT delineate arthritic changes at the midtarsal and TMT joints including joint space narrowing, osteophytes, subchondral sclerosis, and subchondral cysts. On weightbearing films, especially in atraumatic midfoot arthritis, the foot is pronated with low-lying C2 and a negative talometatarsal angle ${ }^{12}$ Lateral films show flattening of the longitudinal arch and collapse of the medial column. The correlation between radiologic signs and clinical symptoms is only moderate. 


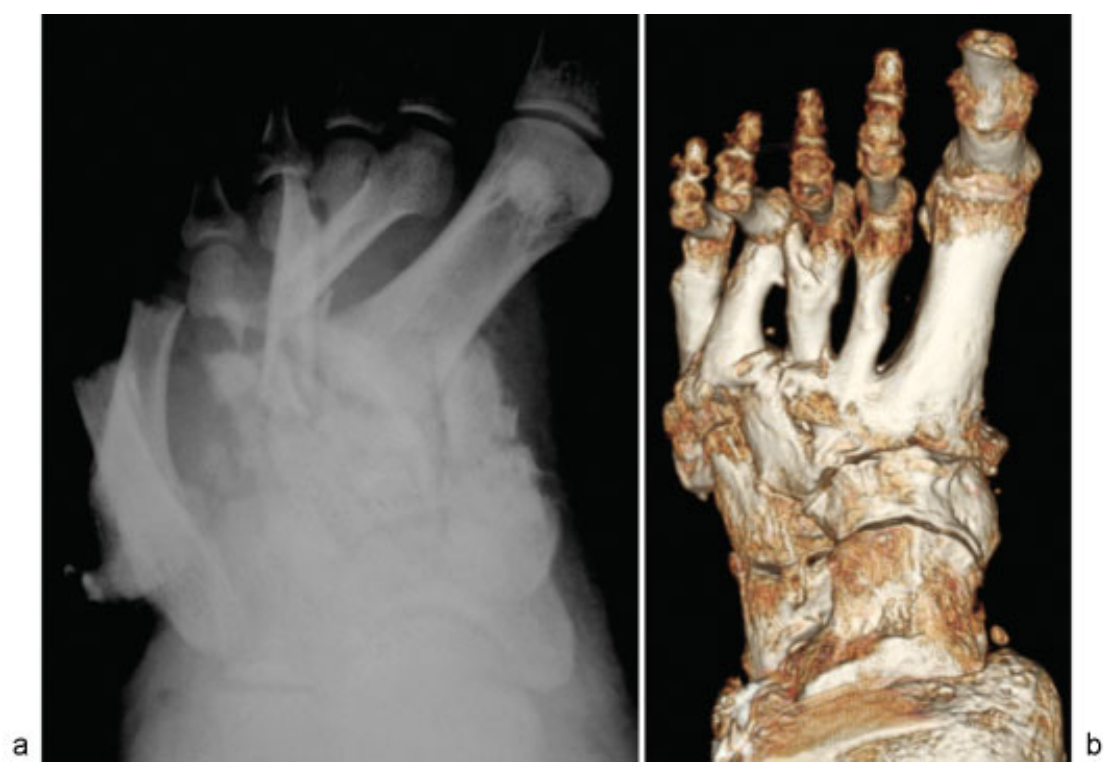

Fig. 30 Complex Lisfranc fracture dislocation (a) with medial and lateral arthrodesis (b).

A combination of anti-inflammatory drugs and orthotic treatment, trying to modify the load to the TMT joints are the first line of treatment ${ }^{12}$ Ultrasound- or CT-guided steroid injections might mitigate pain for some time. ${ }^{24}$ Pain response to injections has been used to test for selecting the joints to fuse; however, the normal communication between the second TMT joint and other joints can be confounding. 12,20,24

Surgery is indicated in patients with refractory pain and restriction of motion after 6 months of nonoperative treatment. Some authors have advocated single-photon emission tomography that combines $\mathrm{CT}$ and bone scan to help in selecting joints for fusion. Surgical options include medial and middle or lateral arthrodesis. Lateral fusion is not recommended unless there is associated collapse because it is a mobile column and motion should be preserved. Lateral column arthrodesis limits walking and increases the risk of pain, nonunion, and stress fracture (-Fig. 28). Surgical complications include infection, peripheral nerve neuromas, nonunion, malunion, persistent pain, and complex regional syndrome. Postoperative metatarsalgia and stress fractures are not uncommon, due to altered biomechanics. $^{12,20,24}$
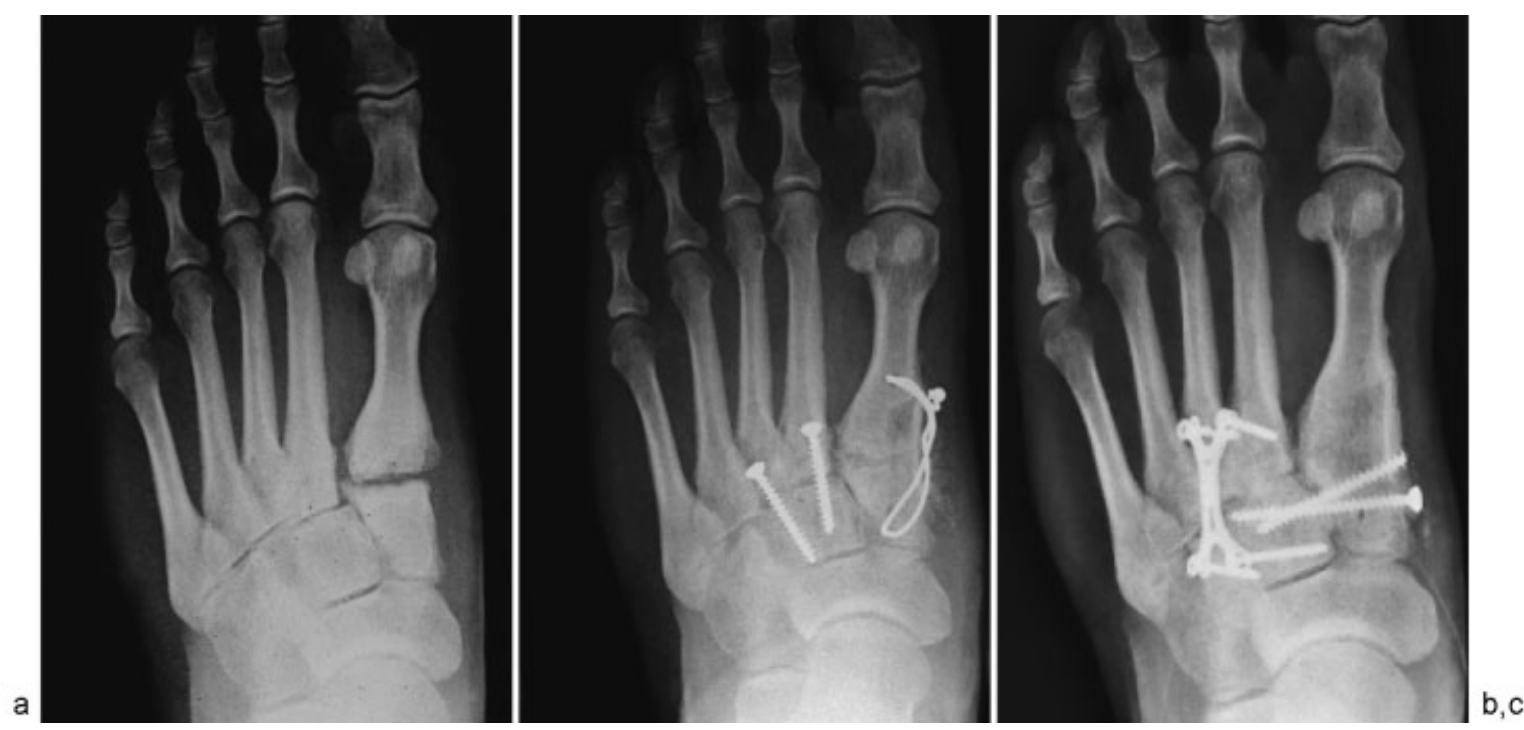

Fig. 31 A 30-year-old patient with a late diagnosis of Lisfranc fracture. (a) Secondary degenerative changes were already present at the time of diagnosis. (b) After initial open reduction and internal fixation of $\mathrm{C} 1-\mathrm{M} 1$ and $\mathrm{C} 2-\mathrm{M} 2-\mathrm{M} 3$, there was continued pain. (c) Middle column arthrodesis was required. 


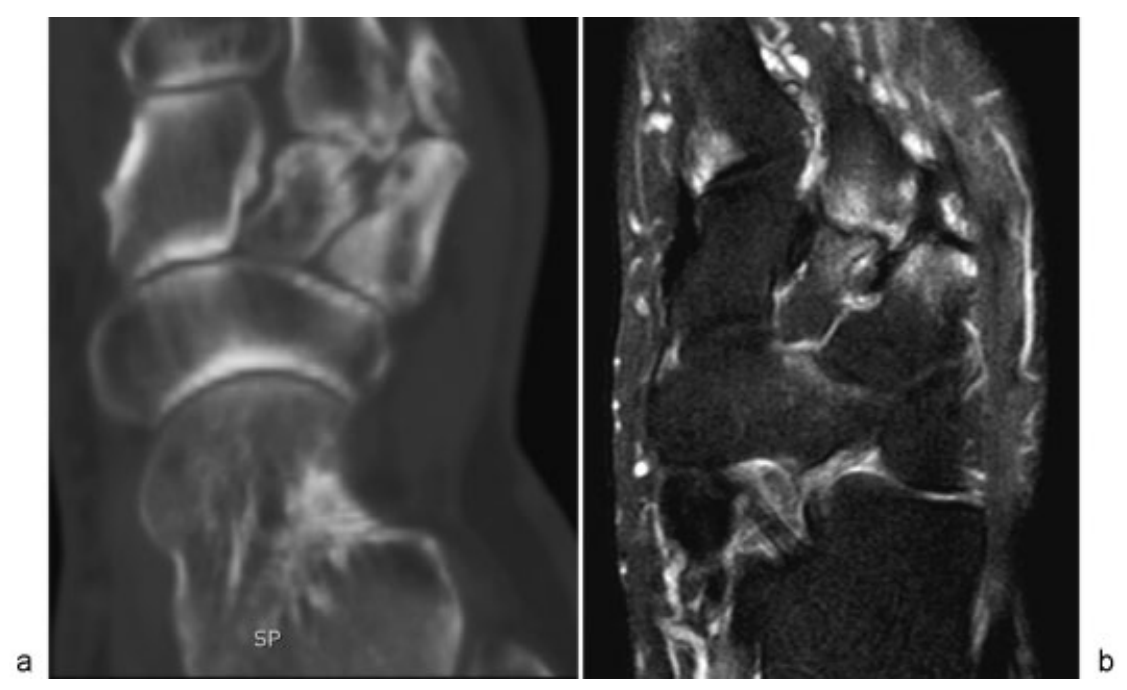

Fig. 32 (a) Axial multidetector computed tomography and (b) axial fat-saturated fast spin-echo T2-weighted image shows posttraumatic intertarsal and tarsometatarsal osteoarthritis due to an undiagnosed Lisfranc fracture.

\section{Acknowledgment}

We thank Elena Belloch, Victoria Higueras, and Joaquin Chismol for their help.

\section{References}

1 Hatem SF. Imaging of Lisfranc injury and midfoot sprain. Radiol Clin North Am 2008;46(6):1045-1060, vi

2 Makwana NK. Tarsometatarsal injuries-Lisfranc injuries. Curr Orthop 2005;19(2):108-118

3 Rosenbaum A, Dellenbaugh S, Dipreta J, Uhl R. Subtle injuries to the Lisfranc joint. Orthopedics 2011;34(11):882-887

4 Gupta RT, Wadhwa RP, Learch TJ, Herwick SM. Lisfranc injury: imaging findings for this important but often-missed diagnosis. Curr Probl Diagn Radiol 2008;37(3):115-126

5 Calder JD, Whitehouse SL, Saxby TS. Results of isolated Lisfranc injuries and the effect of compensation claims. J Bone Joint Surg Br 2004;86(4):527-530

6 de Palma L, Santucci A, Sabetta SP, Rapali S. Anatomy of the Lisfranc joint complex. Foot Ankle Int 1997;18(6):356-364

7 Panchbhavi VK, Molina D IV, Villarreal J, Curry MC, Andersen CR. Three-dimensional, digital, and gross anatomy of the Lisfranc ligament. Foot Ankle Int 2013;34(6):876-880

8 Siddiqui NA, Galizia MS, Almusa E, Omar IM. Evaluation of the tarsometatarsal joint using conventional radiography, CT, and MR imaging. Radiographics 2014;34(2):514-531

9 Umans HR. Imaging sports medicine injuries of the foot and toes. Clin Sports Med 2006;25(4):763-780

10 Castro M, Melão L, Canella C, et al. Lisfranc joint ligamentous complex: MRI with anatomic correlation in cadavers. AJR Am J Roentgenol 2010;195(6):W447-W455

11 Kitsukawa K, Hirano T, Niki H, Tachizawa N, Nakajima Y, Hirata K. MR imaging evaluation of the Lisfranc ligament in cadaveric feet and patients with acute to chronic Lisfranc injury. Foot Ankle Int 2015;36(12):1483-1492
12 Rao S, Nawoczensko D, Baumhauer J. Midfoot arthritis: nonoperative options and decision making for fusion. Tech Foot Ankle Surg 2008;7(3):188-195

13 MacMahon A, Kim P, Levine DS, et al. Return to sports and physical activities after primary partial arthrodesis for Lisfranc injuries in young patients. Foot Ankle Int 2015; November 23 (Epub ahead of print)

14 Early JS, Bucholz RW. Lisfranc injuries and their management. Curr Orthop 1996;10(3):169-173

15 Quénu E, Küss G. Etude sur les luxations du metatarse. Reb Chir 1909;39:281

16 Myerson MS, Fisher RT, Burgess AR, Kenzora JE. Fracture dislocations of the tarsometatarsal joints: end results correlated with pathology and treatment. Foot Ankle 1986;6(5):225-242

17 Curtis MJ, Myerson M, Szura B. Tarsometatarsal joint injuries in the athlete. Am J Sports Med 1993;21(4):497-502

18 Nunley JA, Vertullo CJ. Classification, investigation, and management of midfoot sprains: Lisfranc injuries in the athlete. Am J Sports Med 2002;30(6):871-878

19 Ross G, Cronin R, Hauzenblas J, Juliano P. Plantar ecchymosis sign: a clinical aid to diagnosis of occult Lisfranc tarsometatarsal injuries. J Orthop Trauma 1996;10(2):119-122

20 Verhoeven N, Vandeputte G. Midfoot arthritis: diagnosis and treatment. Foot Ankle Surg 2012;18(4):255-262

21 Faciszewski T, Burks RT, Manaster BJ. Subtle injuries of the Lisfranc joint. J Bone Joint Surg Am 1990;72(10):1519-1522

22 Davies MS, Saxby TS. Intercuneiform instability and the "gap" sign. Foot Ankle Int 1999;20(9):606-609

23 Preidler KW, Peicha G, Lajtai G, et al. Conventional radiography, $\mathrm{CT}$, and MR imaging in patients with hyperflexion injuries of the foot: diagnostic accuracy in the detection of bony and ligamentous changes. AJR Am J Roentgenol 1999;173(6): 1673-1677

24 Drakonaki EE, Kho JS, Sharp RJ, Ostlere SJ. Efficacy of ultrasoundguided steroid injections for pain management of midfoot joint degenerative disease. Skeletal Radiol 2011;40(8):1001-1006 